\title{
Gene expression, proteome and calcium signaling alterations in immortalized hippocampal astrocytes from an Alzheimer's disease mouse model
}

\author{
Francesca Rocchio ${ }^{1,5}$, Laura Tapella', Marcello Manfredii ${ }^{2,3}$, Mariangela Chisari ${ }^{4}$, Francesca Ronco ${ }^{1}$, \\ Federico Alessandro Ruffinatti ${ }^{1}$, Eleonora Conte ${ }^{2}$, Pier Luigi Canonico ${ }^{1}$, Maria Angela Sortino ${ }^{4}$, Mariagrazia Grilli(i) \\ Emilio Marengo ${ }^{2}$, Armando A. Genazzani ${ }^{1}$ and Dmitry Lim (1)
}

\begin{abstract}
Evidence is rapidly growing regarding a role of astroglial cells in the pathogenesis of Alzheimer's disease (AD), and the hippocampus is one of the important brain regions affected in AD. While primary astroglial cultures, both from wildtype mice and from rodent models of $A D$, have been useful for studying astrocyte-specific alterations, the limited cell number and short primary culture lifetime have limited the use of primary hippocampal astrocytes. To overcome these limitations, we have now established immortalized astroglial cell lines from the hippocampus of 3xTg-AD and wildtype control mice (3Tg-iAstro and WT-iAstro, respectively). Both 3Tg-iAstro and WT-iAstro maintain an astroglial phenotype and markers (glutamine synthetase, aldehyde dehydrogenase 1 family member L1 and aquaporin-4) but display proliferative potential until at least passage 25. Furthermore, these cell lines maintain the potassium inward rectifying (Kir) current and present transcriptional and proteomic profiles compatible with primary astrocytes. Importantly, differences between the 3Tg-iAstro and WT-iAstro cell lines in terms of calcium signaling and in terms of transcriptional changes can be re-conducted to the changes previously reported in primary astroglial cells. To illustrate the versatility of this model we performed shotgun mass spectrometry proteomic analysis and found that proteins related to RNA binding and ribosome are differentially expressed in 3Tg-iAstro vs WT-iAstro. In summary, we present here immortalized hippocampal astrocytes from WT and 3xTg-AD mice that might be a useful model to speed up research on the role of astrocytes in $\mathrm{AD}$.
\end{abstract}

\section{Introduction}

While in Alzheimer's disease (AD) astrocytes have been historically associated with reactive gliosis and

\footnotetext{
Correspondence: Armando A. Genazzani (armando.genazzani@uniupo.it) or Dmitry Lim (dmitry.lim@uniupo.it)

${ }^{1}$ Department of Pharmaceutical Sciences, Università degli Studi del Piemonte Orientale, Novara, Italy

${ }^{2}$ Department of Sciences and Technological Innovation, Università degli Studi del Piemonte Orientale, Alessandria, Italy

Full list of author information is available at the end of the article.

These authors contributed equally: Francesca Rocchio, Laura Tapella

Edited by A. Verkhratsky
}

neuroinflammation, a growing body of evidence suggests that astroglial alterations occur in the early stages of $\mathrm{AD}$, compromising their housekeeping and homeostatic functions that in turn may result in synaptic and neuronal malfunction ${ }^{1,2}$.

Most of the information about the role of astrocytes in brain pathology has been collected in in vitro experiments on primary cultures. Easy to prepare and handle, astroglial primary cultures are made from different brain areas ${ }^{3,4}$ and different animal species ${ }^{5-8}$. However, primary cultures present several limitations such as inter-culture 
variability, short culture lifetime and limited number of cells if cultures are prepared from specific brain areas, such as the hippocampus. To overcome these limitations, immortalized astroglial lines have been proposed. The first attempts to generate a permanent astroglial cell line, not deriving from brain tumors, date back to early eighties ${ }^{9}$. Since then, a number of immortalized astroglial lines have been established ${ }^{10-23}$. Most of them derive from a highly heterogeneous population of cortical primary astrocytes; however, immortalization of astrocytes from brain regions other than cortex have also been reported, e.g., from the cerebellum ${ }^{9}$ or from the midbrain ${ }^{21}$. Surprisingly, few attempts have been reported to immortalize astrocytes from hippocampus and also from animal models of $\mathrm{AD}$. In this regard, Morikawa et al. ${ }^{18}$ have generated immortalized astrocytes from ApoE2, ApoE3 and ApoE4 knock-in mice.

In this report we introduce immortalized astroglial lines from the hippocampus of a well-characterized AD mouse model, 3xTg-AD, and from wild-type (WT) control mice, from now on referred to as 3Tg-iAstro and WT-iAstro, respectively. WT-iAstro cell lines show features of primary hippocampal astrocytes such as basic electrophysiological properties, and a similar transcriptional and proteomic profile. More importantly, 3Tg-iAstro show alterations in transcription and deregulation of $\mathrm{Ca}^{2+}$ signaling as it was reported for its primary counterparts. To illustrate the versatility of this model we also performed shotgun mass spectrometry proteomic analysis and found that proteins related to RNA binding and ribosome are differentially expressed in 3Tg-iAstro vs WT-iAstro. These data demonstrate that iAstro lines represent a versatile and useful cellular model to investigate astroglial AD-related pathobiology.

\section{Results}

\section{Generation of immortalized hippocampal astrocytes (iAstro) from WT and 3xTg-AD mice}

Six immortalized cell lines from WT (WT-iAstro\#1-6) and from 3xTg-AD (3Tg-iAstro\#1-6) mice were generated, from separate primary astrocyte cell cultures. For immortalization, primary astroglial cultures were first depleted of microglial cells by magnetic-assisted cell sorting (MACS) using anti-CD11b-conjugated microbeads in order to obtain a population of highly purified astrocytes. Astrocytes were then transduced using retrovirus expressing SV40 large $\mathrm{T}$ antigen. Transformed cells were selected in G418, amplified and stabilized for 12 passages prior to characterization. No clonal selection was performed to maintain the natural hererogeneity of the cultures.

For logistic and experimental setting convenience, four lines for each strain were characterized for morphology and astroglial marker expression. The other two cell lines were confirmed for morphological identity to other lines, but were maintained as backups and have not been characterized (\#1 and \#5 for WT-iAstro and \#1 and \#4 for 3Tg-iAstro lines).

iAstro cells show a morphology similar and virtually indistinguishable to those of primary hippocampal astrocytes in bright field microscopy (Fig. 1a). We also evaluated the expression of the astroglial markers aquaporin-4 (AQP4), glutamine synthetase (GS) and aldehyde dehydrogenase 1 family member 11 (Aldh1l1) (Fig. 1b) by immunocytochemistry. Importantly, we found expression of all three markers in all cell lines. Immunocytochemical analysis for glial fibrillary acidic protein (GFAP) showed, instead, that only a small proportion of cells were positive in the established immortalized cell lines $(15.4 \pm 5.3 \%$ in WT-iAstro vs $16.7 \pm 5.9 \%$ in 3Tg-iAstro cells, $p>0.05$ ) (Fig. 1c), while $100 \%$ of cells were GFAP positive in WTand 3Tg-primary astrocytes.

We also decided to have a quantitative measure of the expression of the three markers by evaluating protein levels in western blotting on three of the four cell lines. The three markers were mostly decreased compared to primary cell lines, albeit at different levels. AQP4 was the least decreased, while Aldh111 was the most decreased, with qualitative consistency in the immortalized cell lines tested. Importantly, not only were all the markers detectable, but they were also represented at similar levels between the WT- and 3Tg-iAstro cell lines (Fig. 2).

We also evaluated the ability of the iAstro cell lines to be passaged in culture. iAstro lines did not change their morphology or marker expression significantly at least up to 20th passage (not shown).

\section{Kir currents are present but are not different in WT-iAstro and $3 \mathrm{Tg}$-iAstro}

Maintenance of ionic balance is one of the housekeeping functions of astrocytes and a feature which might have been lost during immortalization. Potassium buffering by inwardly rectifying $\mathrm{K}^{+}$(Kir) channels during neuronal activity is fundamental to maintain adequate synaptic transmission and neuronal excitability ${ }^{24}$. We have previously shown that Kir channels are functionally expressed in primary hippocampal astrocytes ${ }^{25}$. Therefore, we performed patch-clamp experiments in WTiAstro\#2 and 3Tg-iAstro\#2 lines. To confirm that Kir function was not affected by the immortalization process, control hippocampal mouse astrocytes were also patched in order to record Kir current. Cells were exposed to a step protocol of increasing voltage $(20 \mathrm{mV}$ increments) from $-180 \mathrm{mV}$ to $+60 \mathrm{mV}$ to record current elicited by Kir channels. Before each voltage step increment, cells were kept at $0 \mathrm{mV}$ for $300 \mathrm{~ms}$ in order to block outward potassium flow ${ }^{26}$. Application of this protocol triggered Kir current in the cell lines tested (Fig. 3a). Current (I), 
a
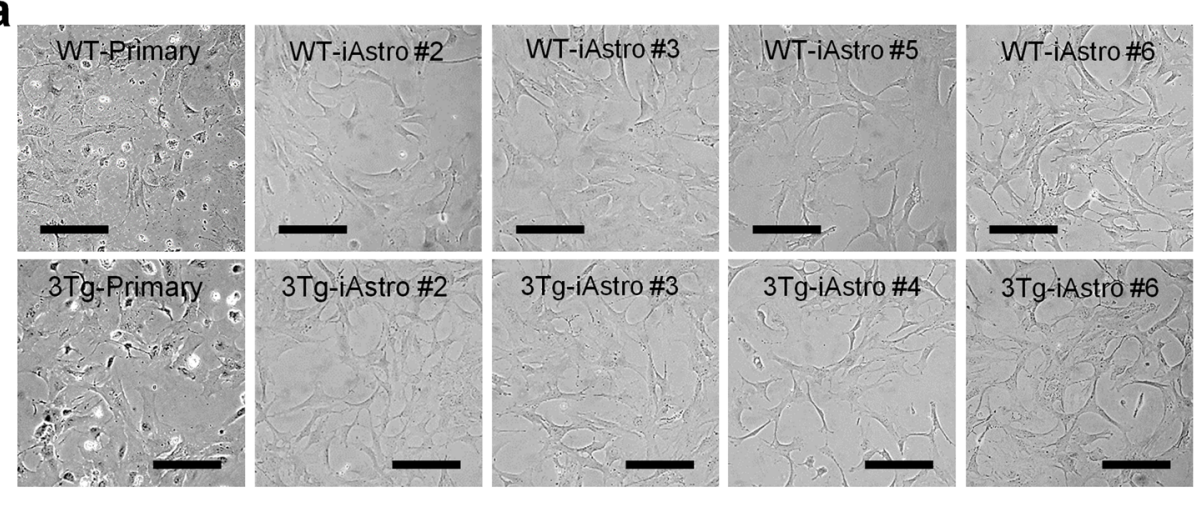

b
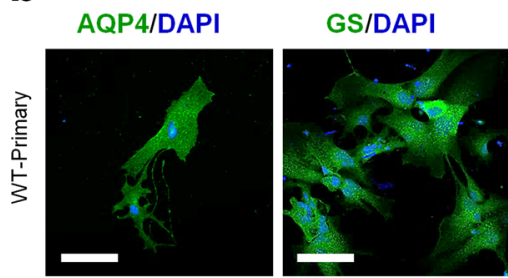

Aldh1/1/DAPI


C
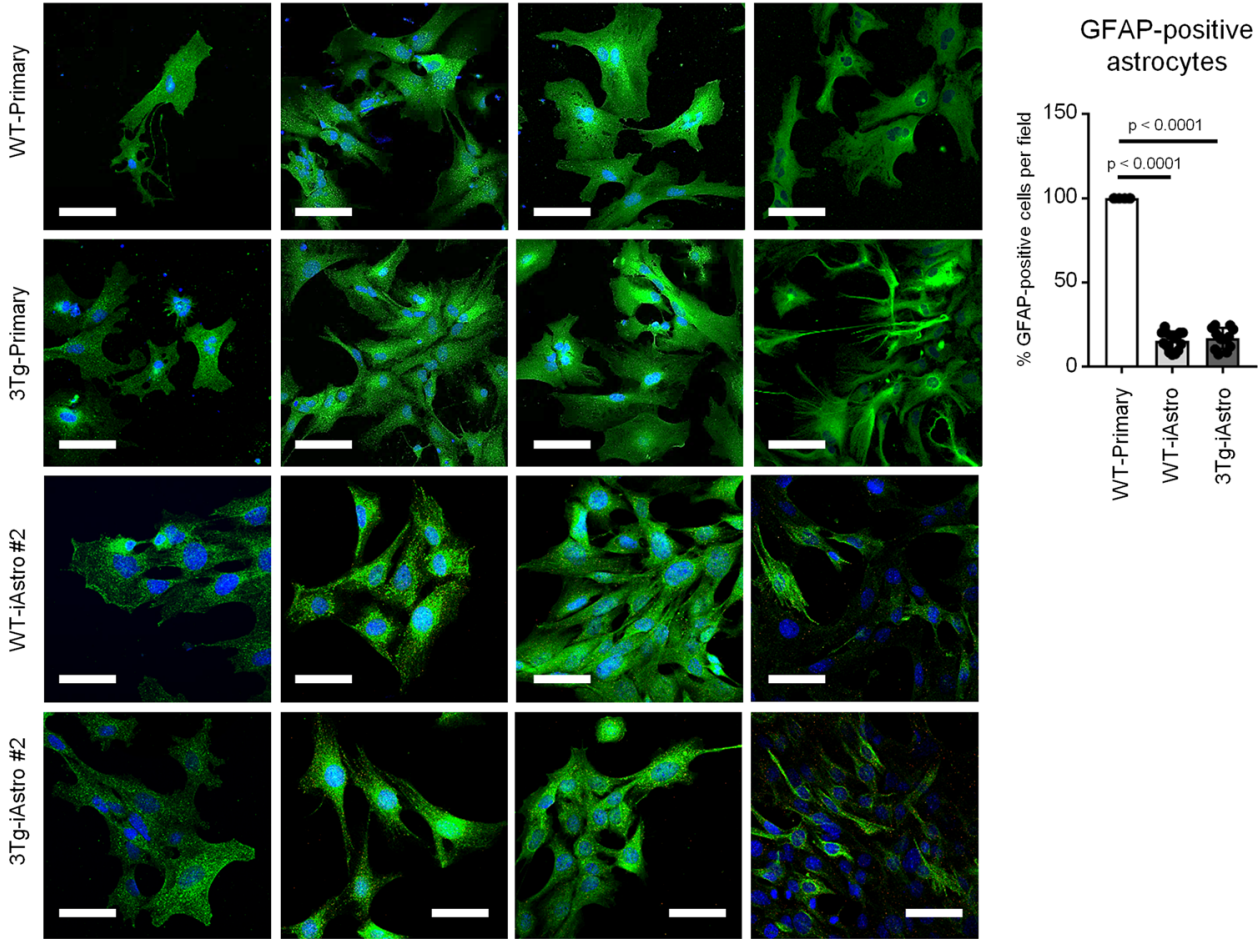

Fig. 1 Characterization of WT- and 3Tg-iAstro lines. a Phase contrast images of four WT-iAstro and four 3Tg-iAstro lines at passage 15. Bar, 100 $\mu \mathrm{m}$. b Immunofluorescence images of WT-iAstro\#2 and 3Tg-iAstro\#2, stained with anti-AQP4, anti-GS, anti-Aldh1l1 and anti-GFAP antibodies. Bar, 50 $\mu \mathrm{m}$. The images shown in $(\mathbf{a}, \mathbf{b})$ are representative of $n=3$ independent experiments. c Quantification of GFAP-positive cells in WT-iAstro\#2 and 3TgiAstro\#2 lines. Data expressed as mean \pm SD \% of 15 fields of GFAP-positive cells evaluating a total of 359 WT- and 5143 Tg-iAstro cells. In (b, c), other characterized independently generated iAstro lines show similar results in immunostaining of astroglial markers and in quantification of GFAPpositive cells (data not shown)

measured at each step of recording protocol, was plotted over corresponding applied voltage $(\mathrm{V})$ to determine $\mathrm{I} / \mathrm{V}$ curve for Kir channels in both cell lines. Raw current values were normalized to maximum I obtained at +60 $\mathrm{mV}$ for each cell (Fig. 3b). No differences were found in iAstro Kir currents through at least five passages. No significant differences were observed in Kir currents between control primary astrocytes and WTand 3Tg-iAstro lines.

\section{iAstro cells are capable of glutamate uptake}

Another fundamental function of astrocytes in the regulation of synaptic transmission is the uptake of glutamate through the action of sodium-dependent glutamate transporters $^{27}$. In the hippocampus, GLT-1 (excitatory amino acid transporter 2 (EAAT2)) is the major glutamate transporter $^{28}$. Therefore, we investigated the expression of GLT1 and glutamate uptake in iAstro lines. As shown in Supplementary Figure 1A, anti-GLT-1 staining revealed 

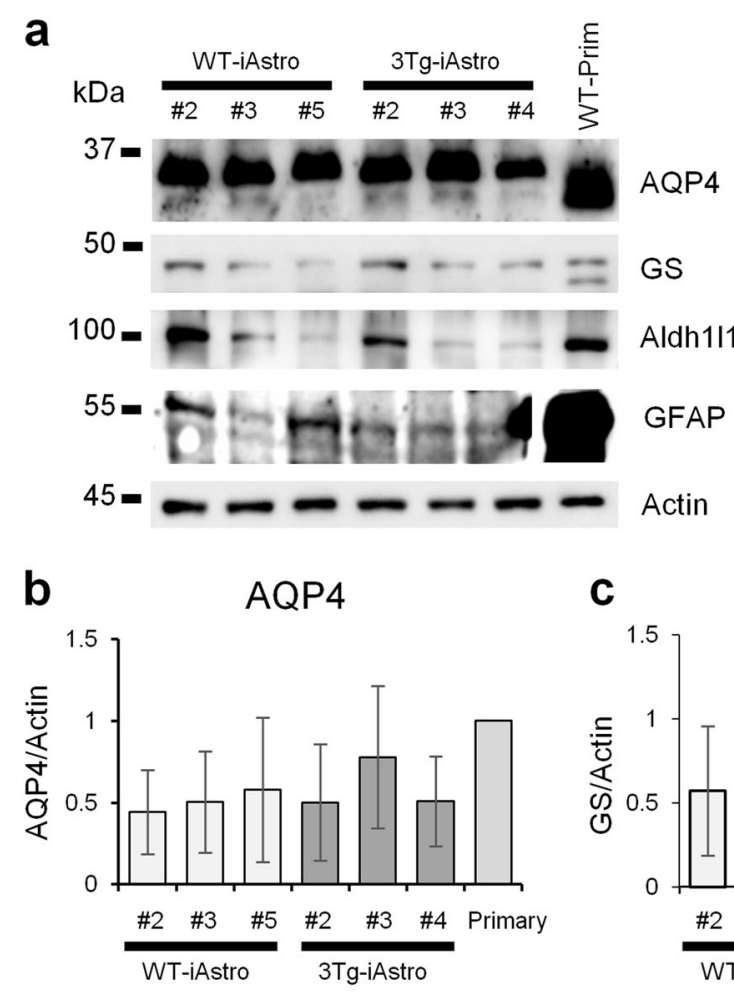

GS

Aldh1l1

GFAP

Actin
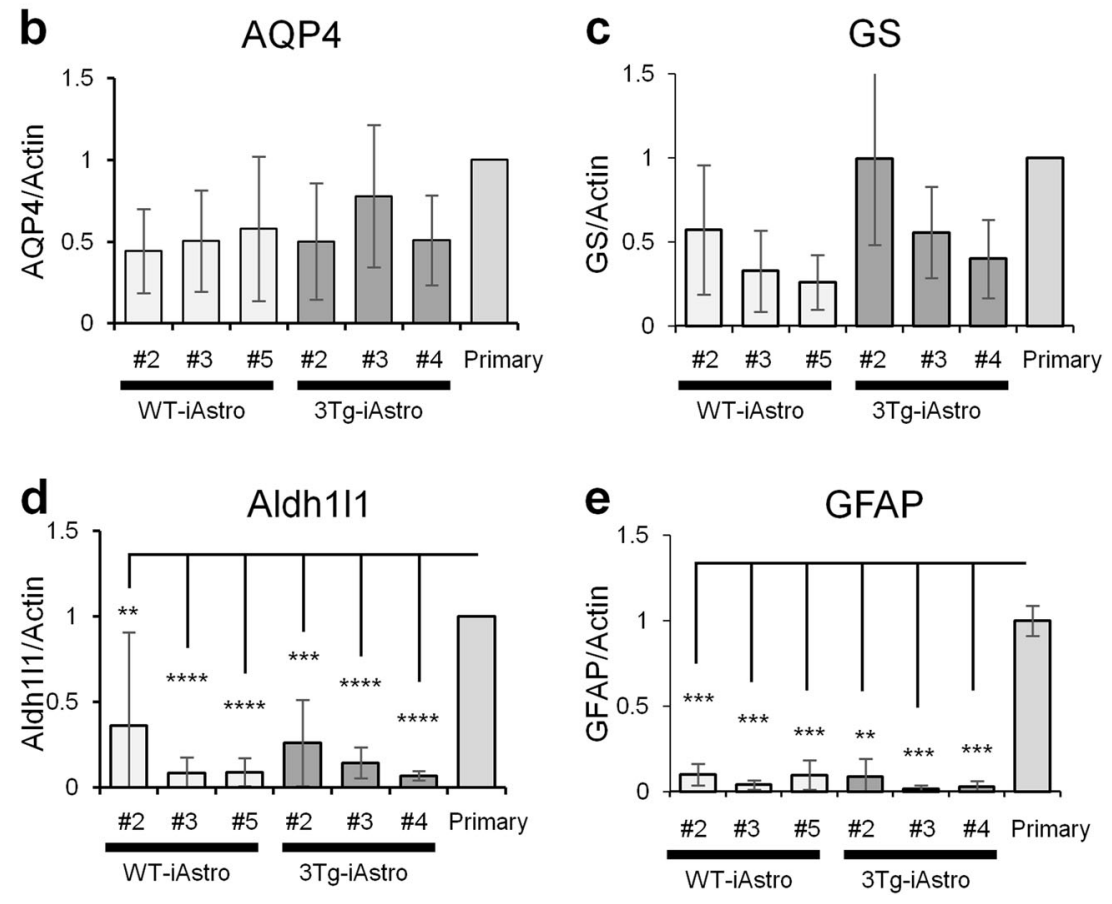

Fig. 2 Western blot analysis and quantification of astroglial marker proteins Aqp4, GS, Aldh1l1 and GFAP. Western blot analysis (a) was performed from four independently generated iAstro lines for each genotype (WT-iAstro\#2, \#3, \#5 and \#6, and 3Tg-iAstro\#2, \#3, \#4 and \#6). Each point represents mean \pm SEM of 3 independent experiments. Actin was used as loading control. ANOVA followed by Tukey's post-hoc test was used for statistical analysis. For Aqp4 (b) and GS (c) there were no significant differences. For Aldh1l1 (d) and GFAP (e) the differences were significant for iAstro lines vs primary astrocytes: ${ }^{* *} p<0.01 ;{ }^{* * *} p<0.001 ;{ }^{* * * *} p<0.0001$

membrane-localized expression of GLT-1 in both primary astrocytes and in iAstro lines. Supplementary Figure 1B shows that WT-iAstro cells are capable of glutamate uptake from the medium with a rate $(10.73 \pm 1.19 \mu \mathrm{mol} / \mathrm{g}$ protein, $n=3)$ comparable to that of primary astrocytes $(19.38 \pm$ $3.74 \mu \mathrm{mol} / \mathrm{g}$ protein, $n=3 ; p=0.092)$. No differences were found in glutamate uptake between WT-iAstro and 3TgiAstro lines (10.77 $\pm 2.14 \mu \mathrm{mol} / \mathrm{g}$ protein, $n=3 ; p=0.12)$.

\section{ATP-induced $\mathrm{Ca}^{2+}$ signaling is altered in immortalized hippocampal astrocytes from 3xTg-AD mice}

Astrocytes respond to external stimuli primarily by generating intracellular $\mathrm{Ca}^{2+}$ signals, employing a combination of release of $\mathrm{Ca}^{2+}$ from the internal stores via metabotropic mechanisms and $\mathrm{Ca}^{2+}$ entry from the extracellular space through the plasma membrane via store-operated $\mathrm{Ca}^{2+}$ entry ${ }^{29-31}$. We have previously shown that astrocytic $\mathrm{Ca}^{2+}$ signaling in $\mathrm{AD}$ is altered using a variety of protocols/models usually employed for neuronal evaluation, including $A \beta$ oligomer treatment ${ }^{32}$, an uncoupling peptide of ADAM10 and SAP97 ${ }^{33}$, or triple transgenic mice ${ }^{34}$. Astrocytic $\mathrm{Ca}^{2+}$ signaling has been shown to be altered in $\mathrm{AD}$ also by others in vivo and in vitro ${ }^{35-38}$.

To validate our immortalized cell lines, we therefore decided to investigate astrocytic $\mathrm{Ca}^{2+}$ signaling using 
Fura-2 single cell $\mathrm{Ca}^{2+}$ imaging. In pilot experiments, 4 WT lines (WT-iAstro\#2, \#3, \#5 and \#6) and 4.3Tg-iAstro lines (3Tg-iAstro\#2, \#3, \#4 and \#6) were tested for adenosine triphosphate (ATP; $20 \mu \mathrm{M}$ )-induced $\mathrm{Ca}^{2+}$ responses, which is among the signaling pathways found altered in $\mathrm{AD}^{34}$. As no significant differences were observed between lines of the same genotype (data not shown), WT-iAstro\#2 and 3Tg-iAstro\#2 were chosen for further analysis. Traces and histograms in Fig. 4a represent mean \pm SEM of 195 cells from WT-iAstro (1.39 \pm 0.035 norm. Fura ratio) and 192 cells from 3Tg-iAstro lines $(1.69 \pm 0.045$ norm. Fura ratio) and show that $3 \mathrm{Tg}$ iAstro exhibited significantly higher amplitude of $\mathrm{Ca}^{2+}$ responses $(p=0.0006)$. As it can be observed, application of ATP to iAstro produces a long-lasting "after-peak" shoulder which is significantly higher in 3Tg-iAstro than in WT-iAstro (48.58 \pm 1.86 area under the curve (AUC) of norm. Fura ratio for WT-iAstro vs $60.83 \pm 1.71$ for 3TgiAstro, $p=0.00005$ ) (Fig. 4c). Given that in primary astrocytes we had observed a similar effect with mGluR5 stimulation that was mediated by store-operated $\mathrm{Ca}^{2+}$ entry (SOCE) ${ }^{39}$, we decided to further explore the effect of ATP. Applications of ATP $(20 \mu \mathrm{M})$ in $\mathrm{Ca}^{2+}$-free solution or pre-treatment with the SOCE inhibitor Pyr3 $(10 \mu \mathrm{M})$ completely eliminated the shoulder in both WTiAstro and 3Tg-iAstro, while the amplitudes of the responses were higher in 3Tg-iAstro cells compared to WT-iAstros (1.31 \pm 0.039 norm. Fura ratio, $n=115$, for 3Tg-iAstro vs $1.19 \pm 0.03, n=118$, for WT-iAstro, $p=$

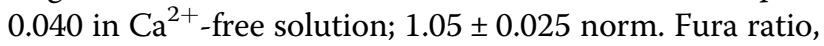
$n=161$, for $3 \mathrm{Tg}$-iAstro vs $0.89 \pm 0.018, n=155$, for WTiAstro, $p=0.000001$ in Pyr3-treated cells) (Fig. 4b). This suggests that ATP, similarly to (RS)-3,5-Dihydroxyphenylglycine (DHPG, an agonist of group I metabotropic glutamate receptors), induced SOCE-mediated $\mathrm{Ca}^{2+}$ entry through the plasma membrane. This was confirmed by re-addition of $\mathrm{Ca}^{2+}$ after depletion of the endoplasmic reticulum $\mathrm{Ca}^{2+}$ stores with a SERCA inhibitor (tBHQ, $20 \mu \mathrm{M}, 5 \mathrm{~min}$ ) in a $\mathrm{Ca}^{2+}$-free Krebs-Ringer modified buffer (KRB) solution (71.5 \pm 1.64 AUC of norm. Fura ratio, $n=66$, for $3 \mathrm{Tg}$-iAstro vs $58.51 \pm 1.94, n=71$, for WT-iAstro, $p=0.00011$ ) (Fig. 4c).

In the current experimental setting the astroglial $\mathrm{Ca}^{2+}$ signals are likely to be mediated by two types of purinergic receptors, P2Y1 and P2Y2. Relative quantification of messenger RNA (mRNA) of P2ry1 and P2ry2 in WT and 3Tg-iAstro lines using real-time PCR showed a higher levels of P2ry2 mRNA compared with P2ry1; moreover, P2ry2 was significantly more expressed in 3Tg-iAstro compared with WT, while levels of P2ry1 were not different. These data suggest that P2Y2 but not P2Y1 may mediate enhanced sensitivity of 3Tg-iAstro to ATP (Fig. 4d).

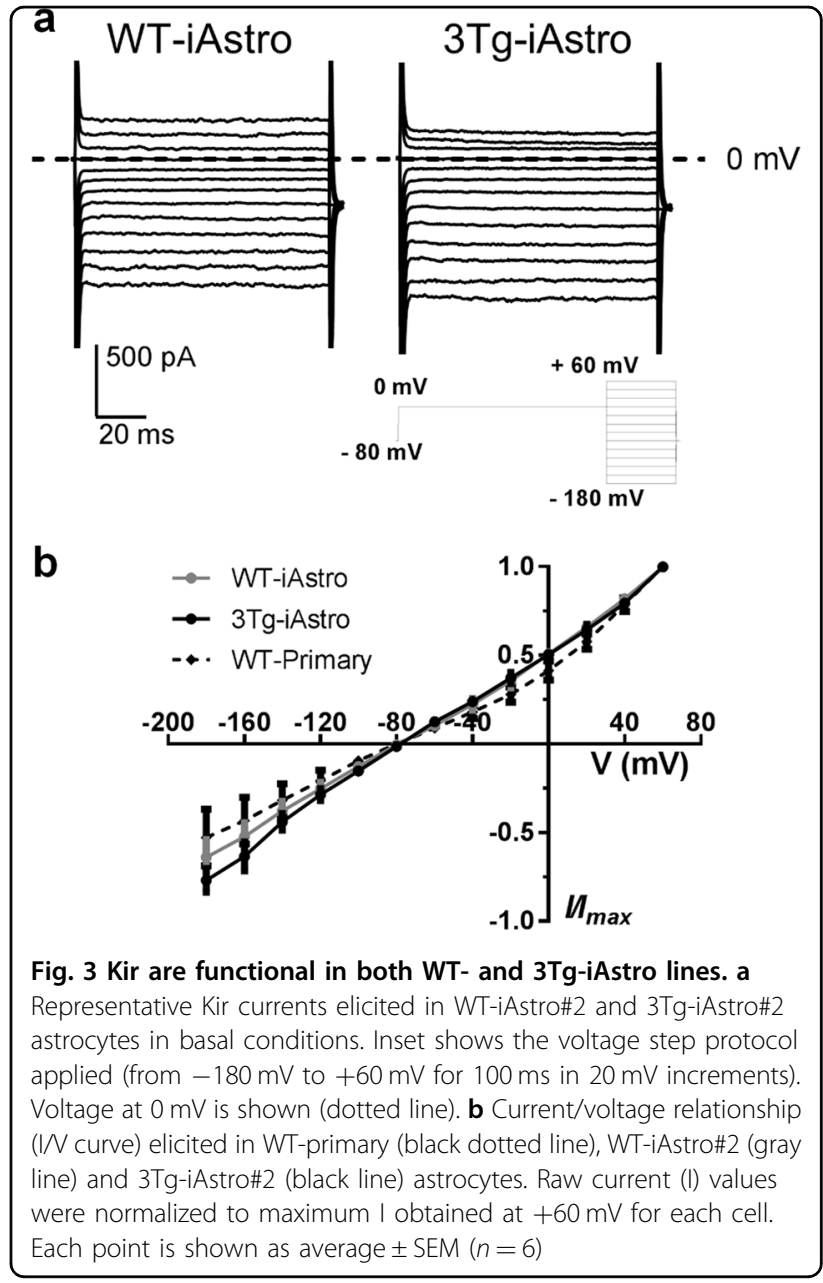

Surprisingly, and contrary to our previous observations on primary astrocytes ${ }^{34}$, we found that iAstro cell lines did not respond to DHPG.

\section{iAstro lines maintain gene expression differences found previously between WT and 3xTg-AD primary astrocytes}

We recently performed a whole-genome microarray analysis of purified primary hippocampal astrocytes ${ }^{40}$ in which bioinformatic analysis revealed expression differences between astrocytes prepared from WT and from $3 \mathrm{Tg}$ animals. To further characterize our immortalized cell lines we therefore primed 14 of the identified genes that showed modifications (Car9, P2ry2, Padi2, Col2a1, Col4a6, Fbln2, Cacna2d3, Panx1, Pcdh17, Trim12, Ccl27a PESKY, Ptchd2, Slc2a4 and Samd4). This set was composed of 6 genes (Pcdh17, Col2a1, Col4a6, Fbln2, Cacna2d3, Panx1) involved in cell adhesion (a gene ontology cluster that was found over-represented), two downregulated genes (Padi2 and Samd4) and a set of 6 up-regulated genes (Trim12, Ptchd2, Slc2a4, Ccl27a PESKY, Car9 and P2ry2). 

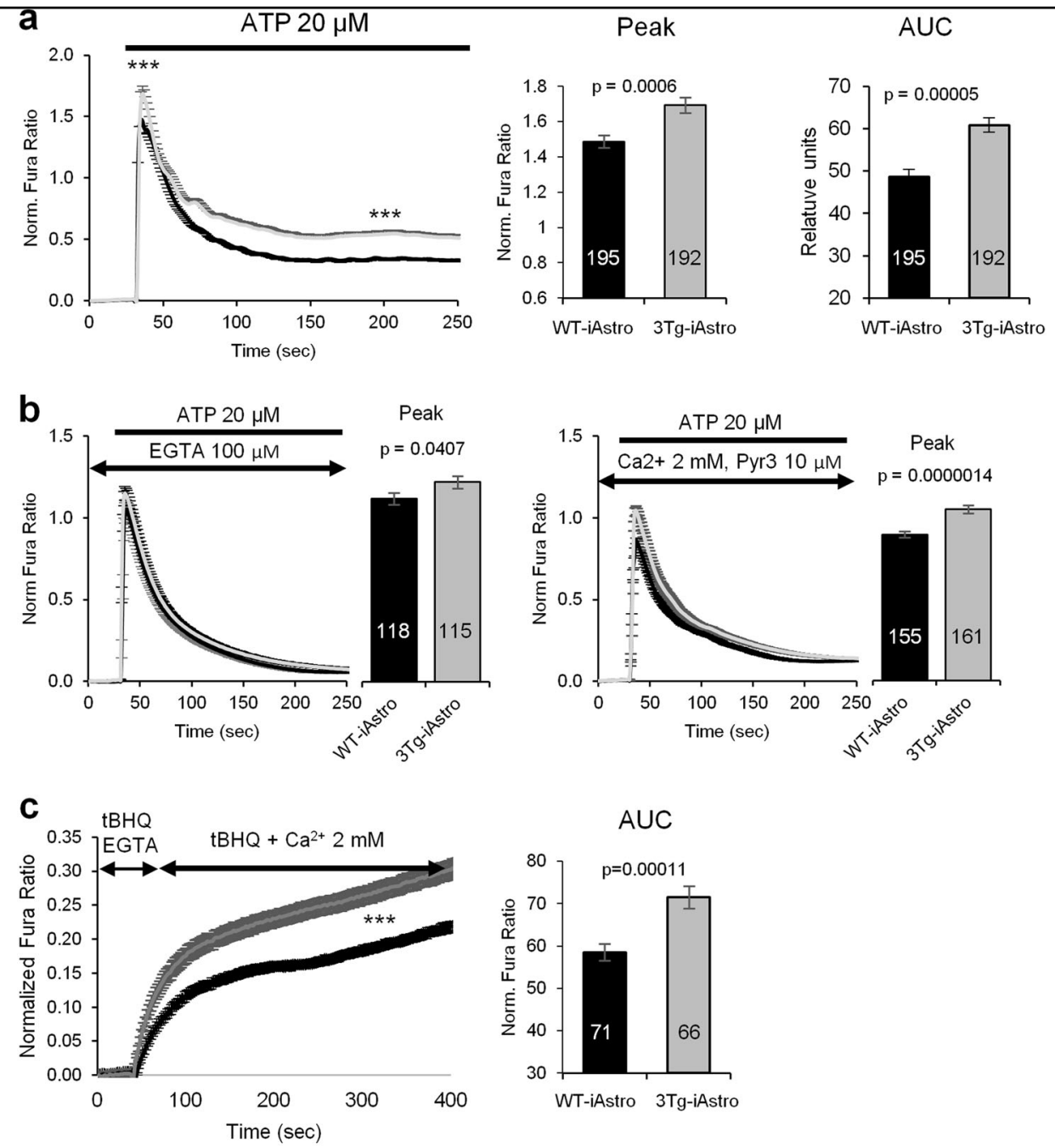

d
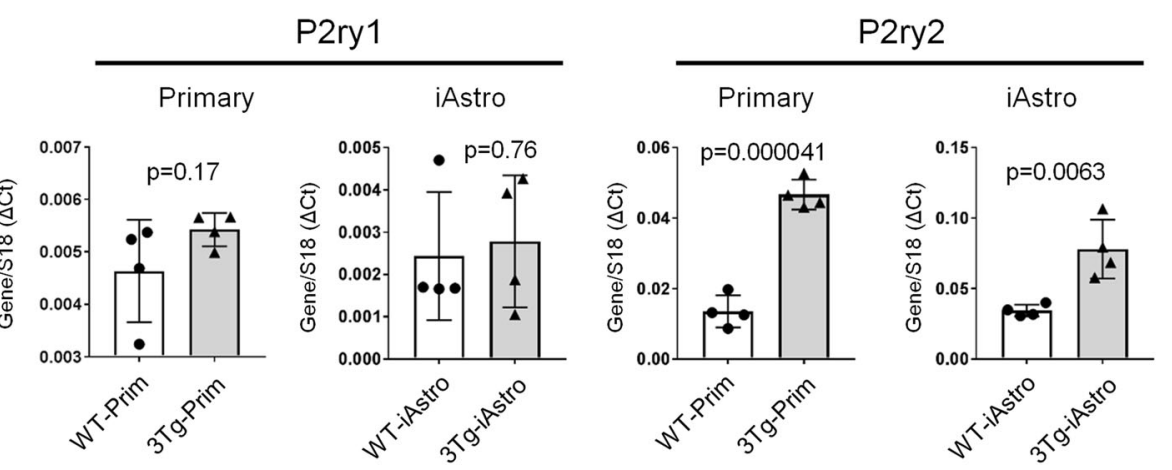

Fig. 4 Calcium signaling alterations in 3Tg-iAstro as compared to WT-iAstro lines. a Fura-2-loaded WT- and 3Tg-iAstro cells were stimulated with $20 \mu \mathrm{M}$ ATP in $\mathrm{Ca}^{2+}$-containing KRB solution. Data are expressed as mean of peak \pm SEM or mean of area under the curve (AUC) \pm SEM of 195 cells (WTiAstro\#2; $1.39 \pm 0.035$ norm. Fura ratio) and 192 cells (3Tg-iAstro\#2; $1.69 \pm 0.045$ norm. Fura ratio, $p=0.0006)$ from 12 coverslips from 3 independent experiments. ${ }^{* *} p<0.001$. b WT- and 3Tg-iAstro cells were stimulated with $20 \mu \mathrm{M}$ ATP in $\mathrm{Ca}^{2+}$-free KRB solution (left panel; mean of peak \pm SEM of 118 cells (WT-iAstro\#2; $1.19 \pm 0.03$ norm. Fura ratio) and 115 cells (3Tg-iAstro\#2; $1.31 \pm 0.039$ norm. Fura ratio, $p=0,040$ ) or in Ca ${ }^{2+}$-containing KRB supplemented with $10 \mu \mathrm{M}$ Pyr3, a store-operated $\mathrm{Ca}^{2+}$ channel blocker (right panel; mean of peak \pm SEM of 155 cells (WT-iAstro\#2; $0.89 \pm 0.018 \mathrm{norm}$. Fura ratio) and 161 cells (3Tg-iAstro\#2; $1.05 \pm 0.025$ norm. Fura ratio, $p=0.000001$ ). c Fura-2-loaded cells were depleted of Ca ${ }^{2+}$ with $20 \mu M$ tBHQ (a SERCA blocker) in $\mathrm{Ca}^{2+}$-free KRB for 5 min after which $\mathrm{Ca}^{2+}$ was re-added and Fura-2 ratio was measured. Data are expressed as mean \pm SEM of AUC of 71 WTiAstro\#2 cells (58.51 \pm 1.94 AUC of norm. Fura ratio) and 66 3Tg-iAstro\#2 cells (71.5 \pm 1.64 AUC of norm. Fura ratio, $p=0.00011)$ from 8 coverslips from 3 independent experiments. d Real-time PCR of P2ry1 (left graphs) and P2ry2 (right graph) from primary astrocytes and iAstro lines. Values represent mean $\pm \mathrm{SD} \Delta \mathrm{C}(\mathrm{t})$ of gene/S18 of four independent cultures. Unpaired two-tailed Student's $t$-test was used for statistical analysis 


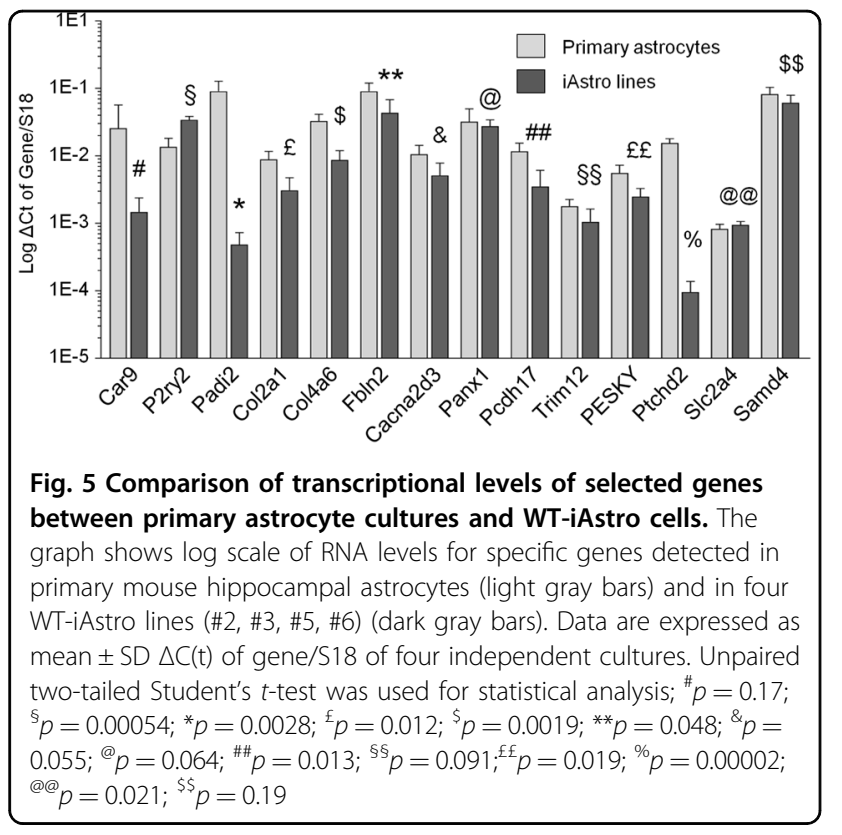

First, we compared levels of the genes between primary WT astrocytes and WT-iAstro, comparing $\Delta \mathrm{C}(\mathrm{t})$ of a gene of interest with respect to $\mathrm{S} 18 \mathrm{C}(\mathrm{t})$. Given the low variability between different primary cultures and between different iAstro cultures, comparison was made averaging data from WT-iAstro \#2, \#3, \#5 and \#6 and four primary cultures. As shown in Fig. 5, most of the genes were expressed in the same order of magnitude between primary and immortalized cells, although a few genes (Car9, Padi2 and Ptchd2) showed a reduced expression of at least one order of magnitude differences.

Our aim, though, was to validate, independently of the expression levels, whether the differences in expression between primary WT and 3Tg astrocytes could be recapitulated in the immortalized cell lines. As shown in Fig. 6a, all 14 genes followed the changes found previously in primary astrocytes ${ }^{40}$. A plot in Fig. 6b shows high degree of correlation between primary and immortalized astrocytes. Taken together, these results show that after the immortalization hippocampal astrocytes retain the transcriptional changes found in primary $3 \mathrm{xTg}$-AD vs WT astrocytes for, at least, a selected group of genes.

\section{Immortalized astrocytes up-regulate iNOS in response to pro-inflammatory stimuli}

The role of astrocytes in neuroinflammation is well ascertained $^{31,41}$, and therefore we have investigated if iAstro lines responded to pro-inflammatory stimuli like bacterial lipopolysaccharide (LPS) or tumor necrosis factor- $\alpha$ (TNF $\alpha)$. As shown in Supplementary Figure 2, inducible nitric oxide synthase (iNOS) was induced upon treatment with both LPS $(100 \mathrm{ng} / \mathrm{ml}$ for $3 \mathrm{~h})$ and TNFa treatment $(20 \mathrm{ng} / \mathrm{ml}$ for $6 \mathrm{~h})$. No differences were found between WT and 3Tg-primary astrocytes or iAstro lines.

We recently reported that transforming growth factor$\beta 2$ (TGF $\beta 2$ ) and TGF $\beta 3$ were up-regulated at mRNA levels in 3Tg-primary astrocytes as compared to WT astroglial cultures ${ }^{42}$. We, therefore, investigated the levels of mRNA of TGF $\beta 2$ and TGF 33 in both non-stimulated and LPS-treated iAstro lines. As shown in Supplementary Figure 3 , in line with cultured primary astrocytes ${ }^{42}$, TGF $\beta 3$ is the most expressed TGF $\beta$ isoform in iAstro lines. Moreover, TGF $\beta 3$ was significantly up-regulated in 3Tg-iAstro $(0.267 \pm 0.02 \Delta \mathrm{C}(\mathrm{t}) \mathrm{TGF} \beta 3 / \mathrm{S} 18, n=4)$ compared to WT-iAsro cells $(0.094 \pm 0.014 \Delta \mathrm{C}(\mathrm{t}) \mathrm{TGF} \beta 3 / \mathrm{S} 18$, $n=4 ; p=0.0012$ ) (Supplementary Figure $3 \mathrm{~B}$ ). No differences were found in TGF $\beta 2$ or TGF $\beta 3$ mRNA levels upon treatment with LPS.

\section{Mass spectrometry proteomics revealed alterations in translation and ribosome in 3Tg-iAstro compared to WT- iAstro}

To further evaluate the iAstro lines, we performed a shotgun mass spectrometry proteomic analysis using the four WT and four 3Tg-iAstro lines. In total, 1119 and 1045 proteins were identified, common to the four analyzed WT- and 3Tg-iAstro lines, respectively (Supplementary Table 1), of which 856 proteins were present in both WT- and 3Tg-iAstro lines (Fig. 7a).

To demonstrate that WT-iAstro cells retain the astrocytic phenotype we compared their proteomic profile with two published datasets obtained through matrix-assisted laser desorption/ionization time-of-flight (MALDI-TOF) mass spectrometry. The first one, provided by Hanrieder et al. $^{43}$, featured 130 unambiguous identified proteins from cultured rat cortical neuroglia. Our dataset included 84 out of 130 (64.6\%) of their entries (see Supplementary Table 2A). Among them, cytochrome $\mathrm{C}$ oxidase (COX5A), cytochrome $\mathrm{C}$ (CYC), ubiquitin (RS27A), chaperonin 10 (CH10), macrophage inhibitor factor (MIF), acetyl co-A binding protein (ACBP), thioredoxin (THIO), calmodulin (CALM), thymosin beta-10 (TYB4) and ribosomal protein S28 (RS28), reported by Hanrieder et al. ${ }^{43}$ as the most astrocyte-specific proteins compared to both oligodendrocytes and microglia, were notably all present in our list. Secondly, we compared iAstro proteins with the proteomic profile provided by Yang et al. ${ }^{44}$, consisting of 178 different proteins from mouse cortical cultured astrocytes. Our list covered 131 out of 178 proteins $(73.6 \%)$ from the dataset provided by Yang et al. ${ }^{44}$ (see Fig. 7a and Supplementary Table 2B). Note a relatively small number of detected proteins in Hanrieder et al. ${ }^{43}$ and Yang et al. ${ }^{44}$ datasets compare to our list of proteins. This may be due to differences in the workflow of the proteome analysis. 


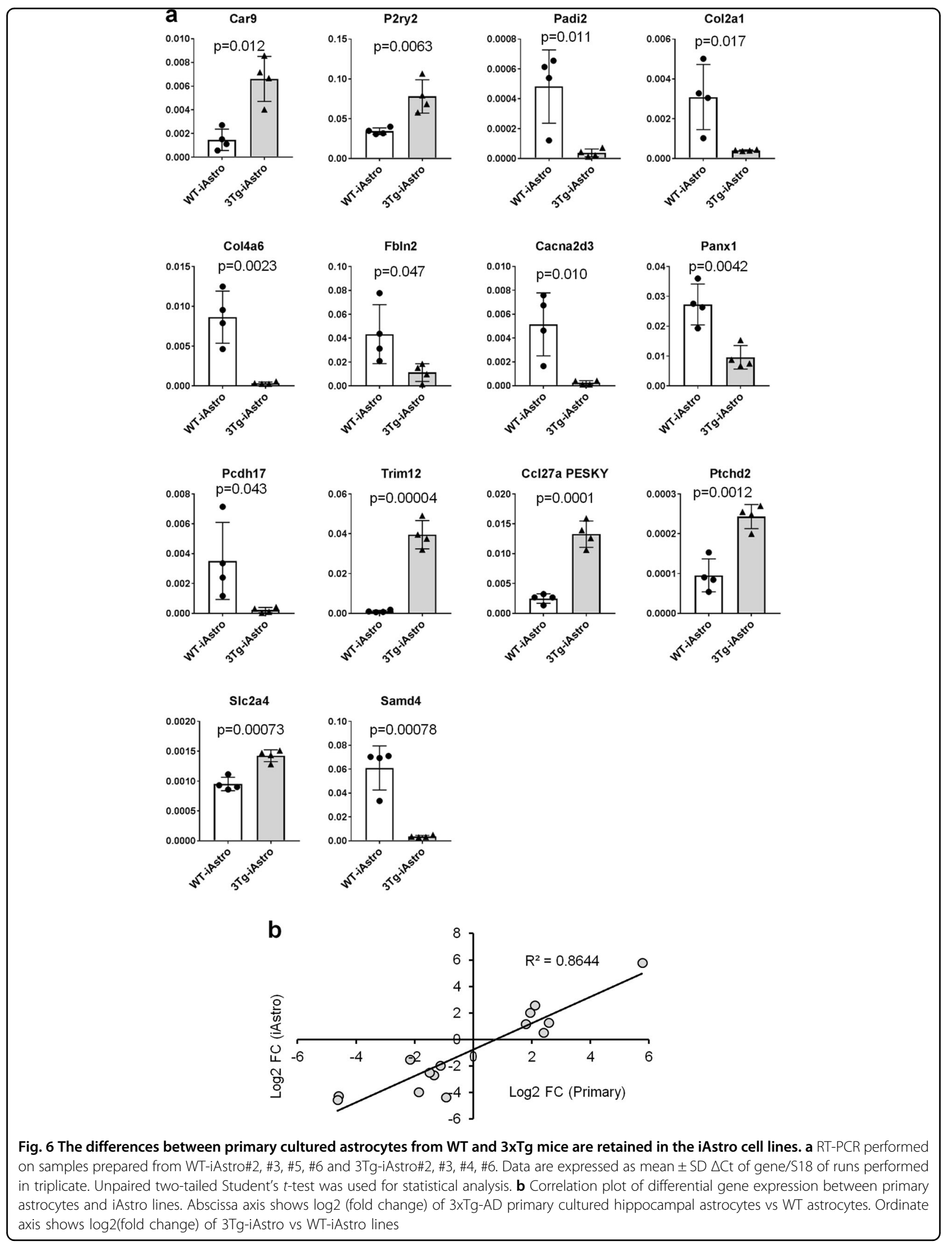




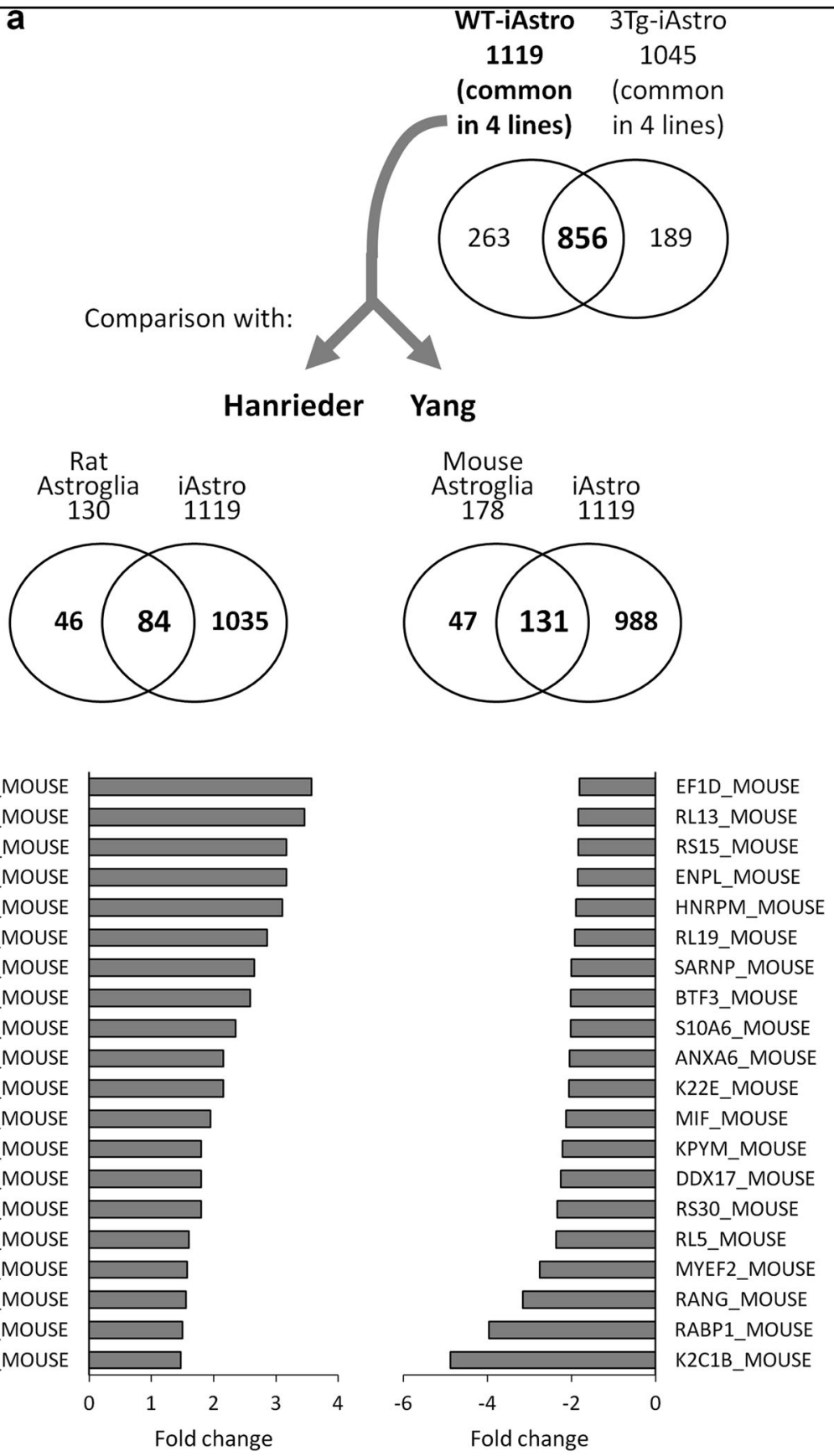

Fig. 7 Mass spectrometry proteomics of differentially expressed proteins. a Protein lysates of WT-iAstro\#2, \#3, \#5, \#6 and 3Tg-iAstro\#2, \#3, \#4,\#6 were subjected to LC-MS/MS with consequent SWATH-MS (see details in Methods section). In total, 1119 and 1045 proteins were detected in WTand 3Tg-iAstro lines, respectively, among which 856 were expressed in both types of samples. The list of 1119 proteins common for all four WT-iAstro lines was compared for presence of common entries with two astroglial proteomics datasets contributed by Hanreider et al. ${ }^{43}$ and Yang et al. ${ }^{44}$. b Top 20 of up- and down-regulated proteins emerged from mass spectrometry proteomics

Next, we performed differential gene expression analysis and identified 73 proteins $(p<0.05$; cut-off $30 \%$ fold change) that were significantly changed between WTiAstro and 3Tg-iAstro, of which 23 were up-regulated and 50 were down-regulated (Fig. 7b, Table 1 and Supplementary Table 3). Functional classification Gene
Ontology (GO) analysis of all 73 differentially expressed proteins using DAVID (Database for Annotation, Visualization and Integrated Discovery) online GO tool returned two groups with significant enrichment score: the first group (enrichment score 12.8) contained 11 proteins all of which were components of ribosome; the 
Table 1 Top 20 up- and down-regulated proteins in 3Tg-iAstro compared to WT-iAstro lines

\begin{tabular}{|c|c|c|c|}
\hline Uniprot_ID & Description & Fold change & $P$ value \\
\hline \multicolumn{4}{|c|}{ Top 20 of UP-regulated proteins } \\
\hline CALM_MOUSE & Calmodulin $\mathrm{OS}=$ Mus musculus $\mathrm{GN}=$ Calm1 $\mathrm{PE}=1 \mathrm{SV}=2$ & 3.57 & 0.00067 \\
\hline ACTG_MOUSE & Actin, cytoplasmic $2 \mathrm{OS}=$ Mus musculus $\mathrm{GN}=\mathrm{Actg} 1 \mathrm{PE}=1 \mathrm{SV}=1$ & 3.46 & 0.00229 \\
\hline EZRI_MOUSE & Ezrin $\mathrm{OS}=$ Mus musculus $\mathrm{GN}=\mathrm{Ezr} \mathrm{PE}=1 \mathrm{SV}=3$ & 3.16 & 0.00189 \\
\hline PUR4_MOUSE & Phosphoribosylformylglycinamidine synthase $\mathrm{OS}=$ Mus musculus $\mathrm{GN}=\mathrm{Pfas} \mathrm{PE}=2 \mathrm{SV}=1$ & 3.16 & 0.00287 \\
\hline ACTB_MOUSE & Actin, cytoplasmic $1 \mathrm{OS}=$ Mus musculus $\mathrm{GN}=\mathrm{Actb} \mathrm{PE}=1 \mathrm{SV}=1$ & 3.10 & 0.00358 \\
\hline MAOM_MOUSE & NAD-dependent malic enzyme, mitochondrial OS $=$ Mus musculus $G N=$ Me2 $P E=2 S V=1$ & 2.86 & 0.01149 \\
\hline APMAP_MOUSE & Adipocyte plasma membrane-associated protein $\mathrm{OS}=$ Mus musculus $\mathrm{GN}=$ Apmap $\mathrm{PE}=1 \mathrm{SV}=1$ & 2.66 & 0.04597 \\
\hline VDAC1_MOUSE & Isoform Mt-VDAC1 of Voltage-dependent anion-selective channel protein $1 \mathrm{OS}=$ Mus musculus GN = Vdac1 & 2.58 & 0.04867 \\
\hline MYH10_MOUSE & Myosin-10 OS $=$ Mus musculus GN $=$ Myh10 PE $=1 \mathrm{SV}=2$ & 2.35 & 0.01125 \\
\hline XPO1_MOUSE & Exportin-1 OS $=$ Mus musculus GN $=X \mathrm{Xpo} 1 \mathrm{PE}=1 \mathrm{SV}=1$ & 2.16 & 0.01711 \\
\hline MYADM_MOUSE & Myeloid-associated differentiation marker $\mathrm{OS}=$ Mus musculus $\mathrm{GN}=$ Myadm $\mathrm{PE}=2 \mathrm{SV}=2$ & 2.15 & 0.03671 \\
\hline MCA3_MOUSE & Eukaryotic translation elongation factor 1 epsilon-1 OS $=$ Mus musculus GN $=$ Eef1e1 PE $=2 \mathrm{SV}=1$ & 1.95 & 0.03302 \\
\hline KCRB_MOUSE & Creatine kinase B-type $\mathrm{OS}=$ Mus musculus $\mathrm{GN}=\mathrm{Ckb} P E=1 \mathrm{SV}=1$ & 1.80 & 0.04875 \\
\hline HMOX1_MOUSE & Heme oxygenase $1 \mathrm{OS}=$ Mus musculus $\mathrm{GN}=\mathrm{Hmox} 1 \mathrm{PE}=1 \mathrm{SV}=1$ & 1.80 & 0.01236 \\
\hline FERM2_MOUSE & Fermitin family homolog $2 \mathrm{OS}=$ Mus musculus $\mathrm{GN}=$ Fermt2 $\mathrm{PE}=1 \mathrm{SV}=1$ & 1.80 & 0.02534 \\
\hline GLRX3_MOUSE & Glutaredoxin-3 OS $=$ Mus musculus GN $=\mathrm{Glr} \times 3 \mathrm{PE}=1 \mathrm{SV}=1$ & 1.60 & 0.03293 \\
\hline ADT1_MOUSE & ADP/ATP translocase $1 \mathrm{OS}=$ Mus musculus $\mathrm{GN}=\mathrm{SIc} 25 \mathrm{a} 4 \mathrm{PE}=1 \mathrm{SV}=4$ & 1.58 & 0.00764 \\
\hline IF4H_MOUSE & Eukaryotic translation initiation factor $4 \mathrm{H} \mathrm{OS}=$ Mus musculus $\mathrm{GN}=\mathrm{Eif} 4 \mathrm{~h} \mathrm{PE}=1 \mathrm{SV}=3$ & 1.55 & 0.00594 \\
\hline IF5A1_MOUSE & Eukaryotic translation initiation factor $5 \mathrm{~A}-1 \mathrm{OS}=$ Mus musculus $\mathrm{GN}=\mathrm{Eif5}$ a $\mathrm{PE}=1 \mathrm{SV}=2$ & 1.50 & 0.03688 \\
\hline FSCN1_MOUSE & Fascin $\mathrm{OS}=$ Mus musculus GN $=F s c n 1 P E=1 \mathrm{SV}=4$ & 1.47 & 0.02807 \\
\hline \multicolumn{4}{|c|}{ Top 20 of DOWN-regulated proteins } \\
\hline K2C1B_MOUSE & Keratin, type II cytoskeletal $1 \mathrm{~b}$ OS $=$ Mus musculus GN $=$ Krt77 PE $=1 \mathrm{SV}=1$ & -4.89 & 0.02491 \\
\hline RABP1_MOUSE & Cellular retinoic acid-binding protein $1 \mathrm{OS}=$ Mus musculus $\mathrm{GN}=\mathrm{Crabp} 1 \mathrm{PE}=1 \mathrm{SV}=2$ & -3.96 & 0.00811 \\
\hline RANG_MOUSE & Ran-specific GTPase-activating protein OS $=$ Mus musculus $\mathrm{GN}=$ Ranbp1 $\mathrm{PE}=1 \mathrm{SV}=2$ & -3.15 & 0.000058 \\
\hline MYEF2_MOUSE & Myelin expression factor $2 \mathrm{OS}=$ Mus musculus $\mathrm{GN}=$ Myef2 $\mathrm{PE}=1 \mathrm{SV}=1$ & -2.76 & 0.00512 \\
\hline RL5_MOUSE & $60 \mathrm{~S}$ ribosomal protein $\mathrm{L} 5 \mathrm{OS}=$ Mus musculus $\mathrm{GN}=\mathrm{Rp} 15 \mathrm{PE}=1 \mathrm{SV}=3$ & -2.36 & 0.00058 \\
\hline RS30_MOUSE & $40 \mathrm{~S}$ ribosomal protein $\mathrm{S} 30 \mathrm{OS}=$ Mus musculus $\mathrm{GN}=\mathrm{Fau} P E=1 \mathrm{SV}=1$ & -2.33 & 0.01976 \\
\hline DDX17_MOUSE & Probable ATP-dependent RNA helicase DDX17 OS $=$ Mus musculus GN $=$ Ddx17 PE $=1 \mathrm{SV}=1$ & -2.25 & 0.0443 \\
\hline KPYM_MOUSE & Isoform M1 of pyruvate kinase PKM OS = Mus musculus GN = Pkm & -2.21 & 0.01086 \\
\hline MIF_MOUSE & Macrophage migration inhibitory factor $\mathrm{OS}=$ Mus musculus $\mathrm{GN}=$ Mif $\mathrm{PE}=1 \mathrm{SV}=2$ & -2.13 & 0.04215 \\
\hline K22E_MOUSE & Keratin, type $\|$ cytoskeletal 2 epidermal $\mathrm{OS}=$ Mus musculus $\mathrm{GN}=\mathrm{Krt} 2 \mathrm{PE}=1 \mathrm{SV}=1$ & -2.06 & 0.04617 \\
\hline ANXA6_MOUSE & Annexin $\mathrm{A} 6 \mathrm{OS}=$ Mus musculus $\mathrm{GN}=$ Anxa6 $\mathrm{PE}=1 \mathrm{SV}=3$ & -2.05 & 0.01972 \\
\hline S10A6_MOUSE & Protein S100-A6 OS $=$ Mus musculus GN $=\mathrm{S} 100 \mathrm{a} 6 \mathrm{PE}=1 \mathrm{SV}=3$ & -2.02 & 0.00021 \\
\hline BTF3_MOUSE & Isoform 2 of transcription factor BTF3 OS $=$ Mus musculus $\mathrm{GN}=\mathrm{Btf3}$ & -2.02 & 0.00144 \\
\hline SARNP_MOUSE & SAP domain-containing ribonucleoprotein $\mathrm{OS}=$ Mus musculus $\mathrm{GN}=$ Sarnp $\mathrm{PE}=1 \mathrm{SV}=3$ & -2.01 & 0.03162 \\
\hline RL19_MOUSE & $60 \mathrm{~S}$ ribosomal protein $\mathrm{L} 19 \mathrm{OS}=$ Mus musculus $\mathrm{GN}=\mathrm{Rp} 19 \mathrm{PE}=1 \mathrm{SV}=1$ & -1.92 & 0.0261 \\
\hline HNRPM_MOUSE & Heterogeneous nuclear ribonucleoprotein $\mathrm{M} O S=$ Mus musculus $\mathrm{GN}=\mathrm{Hnrnpm} \mathrm{PE}=1 \mathrm{SV}=3$ & -1.89 & 0.00045 \\
\hline ENPL_MOUSE & Endoplasmin OS $=$ Mus musculus GN $=$ Hsp90b1 PE $=1 \mathrm{SV}=2$ & -1.85 & 0.01527 \\
\hline RS15_MOUSE & $40 \mathrm{~S}$ ribosomal protein $\mathrm{S} 15 \mathrm{OS}=$ Mus musculus $\mathrm{GN}=\mathrm{Rps} 15 \mathrm{PE}=2 \mathrm{SV}=2$ & -1.84 & 0.00744 \\
\hline RL13_MOUSE & $60 \mathrm{~S}$ ribosomal protein $\mathrm{L} 13 \mathrm{OS}=$ Mus musculus $\mathrm{GN}=\mathrm{Rpl} 13 \mathrm{PE}=2 \mathrm{SV}=3$ & -1.83 & 0.03011 \\
\hline EF1D_MOUSE & Elongation factor 1-delta $\mathrm{OS}=$ Mus musculus $\mathrm{GN}=$ Eef1d $\mathrm{PE}=1 \mathrm{SV}=3$ & -1.80 & 0.03256 \\
\hline
\end{tabular}

second group (enrichment score 8.9) was composed of 5 proteins related to RNA binding and to the formation of ribonucleoprotein complex (Supplementary Table 4A). To explore functional significance of up-regulated vs down-regulated proteins, we analyzed separately 23 upregulated and 50 down-regulated proteins. Analysis of up-regulated proteins did not return significantly overrepresented GO terms, while functional annotation GO analysis of the 50 down-regulated proteins returned 10 significantly overrepresented GO terms which were related to RNA binding, ribonucleoprotein complex, ribosome and nucleus (Table 2 and Supplementary Table 4B).

We then used STRING (Search Tool for the Retrieval of Interacting Genes/Proteins) database that allows prediction of protein-protein interacting networks and clustering. For this we used a list of 73 differentially expressed proteins which included both down- and up-regulated hits. As shown in Fig. 8, STRING software found significantly more interactions than may be expected by 
Table 2 Over-represented GO terms

\begin{tabular}{|c|c|c|c|c|}
\hline GO category & GO term & Count & Fold enrichment & Benjamini \\
\hline GOTERM_CC_DIRECT & GO:0030529 intracellular ribonucleoprotein complex & 17 & 3.30 & 0.0013 \\
\hline GOTERM_CC_DIRECT & GO:0005840 ribosome & 12 & 3.96 & 0.0051 \\
\hline GOTERM_CC_DIRECT & GO:0005634 nucleus & 37 & 1.51 & 0.0121 \\
\hline GOTERM_CC_DIRECT & GO:0022625 cytosolic large ribosomal subunit & 8 & 4.42 & 0.0391 \\
\hline GOTERM_MF_DIRECT & GO:0044822 poly(A) RNA binding & 31 & 2.25 & 0.0001 \\
\hline GOTERM_MF_DIRECT & GO:0003735 structural constituent of ribosome & 12 & 3.54 & 0.0191 \\
\hline GOTERM_MF_DIRECT & GO:0003723 RNA binding & 16 & 2.37 & 0.0577 \\
\hline KEGG_PATHWAY & mmu03010:Ribosome & 12 & 3.56 & 0.0065 \\
\hline UP_KEYWORDS & Ribonucleoprotein & 15 & 3.03 & 0.0074 \\
\hline UP_KEYWORDS & Ribosomal protein & 12 & 3.77 & 0.0146 \\
\hline
\end{tabular}

chance $(p<1.0 \mathrm{e}-16)$. To search for possible interacting clusters we used STRING $k$-means clustering function followed by $\mathrm{GO}$ analysis. Figure 8 shows three clusters found by STRING. GO analysis revealed that cluster \#1 (red in Fig. 8) returned two GO terms, myelin sheath and extracellular exosome. Cluster \#2 (green in Fig. 8) returned $13 \mathrm{GO}$ terms related to translation, ribosome and RNA binding, while cluster \#3 (blue in Fig. 8) returned 15 GO terms related to nuclear ribonucleoprotein complex and splicing (Supplementary Table 5). Altogether, this analysis suggests that in 3Tg-iAstro lines, the protein synthesis machinery may be impaired.

\section{Discussion}

Here we report the generation and characterization of novel immortalized astroglial lines from the hippocampus of a common $\mathrm{AD}$ mouse model, $3 \times \mathrm{Tg}-\mathrm{AD}$ mice ${ }^{45}$ and from its WT counterpart. For immortalization, we used transduction with SV40 large T antigen, a protocol that has been extensively used previously ${ }^{14,46,47}$. During exploitation of the protocol we did not proceed with clonal selection, but instead continued growing and expanding a population of transduced cells. This allowed us to avoid the inter-clonal heterogeneity that is a characteristic of clonal selection ${ }^{48-50}$. Validity of this approach was efficiently demonstrated in gene expression and proteomics analyses, in which four independently generated iAstro lines for both WT and 3Tg-AD genotypes were analyzed and gave consistent results, and also demonstrated that 3Tg-iAstro retains the differences as compared with WT-iAstro found previously in primary cultures $^{40}$.

To validate our model we used a number of methods including immunocytochemistry, real-time PCR (RT-PCR), electrophysiology and $\mathrm{Ca}^{2+}$ measurements. We show that the WT-iAstro cell lines express the astrocytic markers AQP4, GS and Aldh111, although at lower levels compared to primary cells and have electrophysiological properties comparable to primary astrocytes, at least as determined by evaluation of the Kir current. Similarly, real-time PCR of selected genes as well as mass spectrometry for proteins shows that the expression profile is similar to primary astrocytes. For a quantitative estimation of the astrocytic phenotype of iAstro lines, we have compared our proteomics data with similar data obtained on primary astroglial cultures. For this we used two lists reporting data from primary cultured rat $^{43}$ and mouse ${ }^{44}$ cortical astrocytes. When our list was compared with the above-mentioned datasets, relatively high percentage of common proteins was found (65\% of those reported by Hanrieder et al. ${ }^{43}$ and $74 \%$ of those reported by Yang et al. $^{44}$ ), indicating that our list efficiently covers both analyzed datasets. This further confirms that after immortalization procedure, iAstro retains an astroglial phenotype. The only difference of note observed was a small proportion of GFAP-positive cells.

Having established that iAstro cell lines replicate primary culture, we next evaluated whether the changes observed in AD models could be replicated by the 3xTgiAstro cells. For this, we capitalized on our previous data on $\mathrm{Ca}^{2+}$ signaling and on transcriptional profiling. Deregulations of astroglial $\mathrm{Ca}^{2+}$ signaling in $\mathrm{AD}$ have been reported ${ }^{30,51-53}$. Our group has demonstrated that primary hippocampal astrocytes from 3xTg-AD mice exhibit enhanced ATP-induced $\mathrm{Ca}^{2+}$ signals ${ }^{34}$ and $\mathrm{SOCE}^{39}$. In line with results on primary cultures, 3TgiAstro exhibited significantly higher amplitude of $\mathrm{Ca}^{2+}$ signals in response to ATP stimulation which is accompanied by enhanced SOCE. Analyzing components of the purinergic $\mathrm{Ca}^{2+}$ signaling we noticed that P2ry2 ATPsensitive receptor is significantly up-regulated in both 


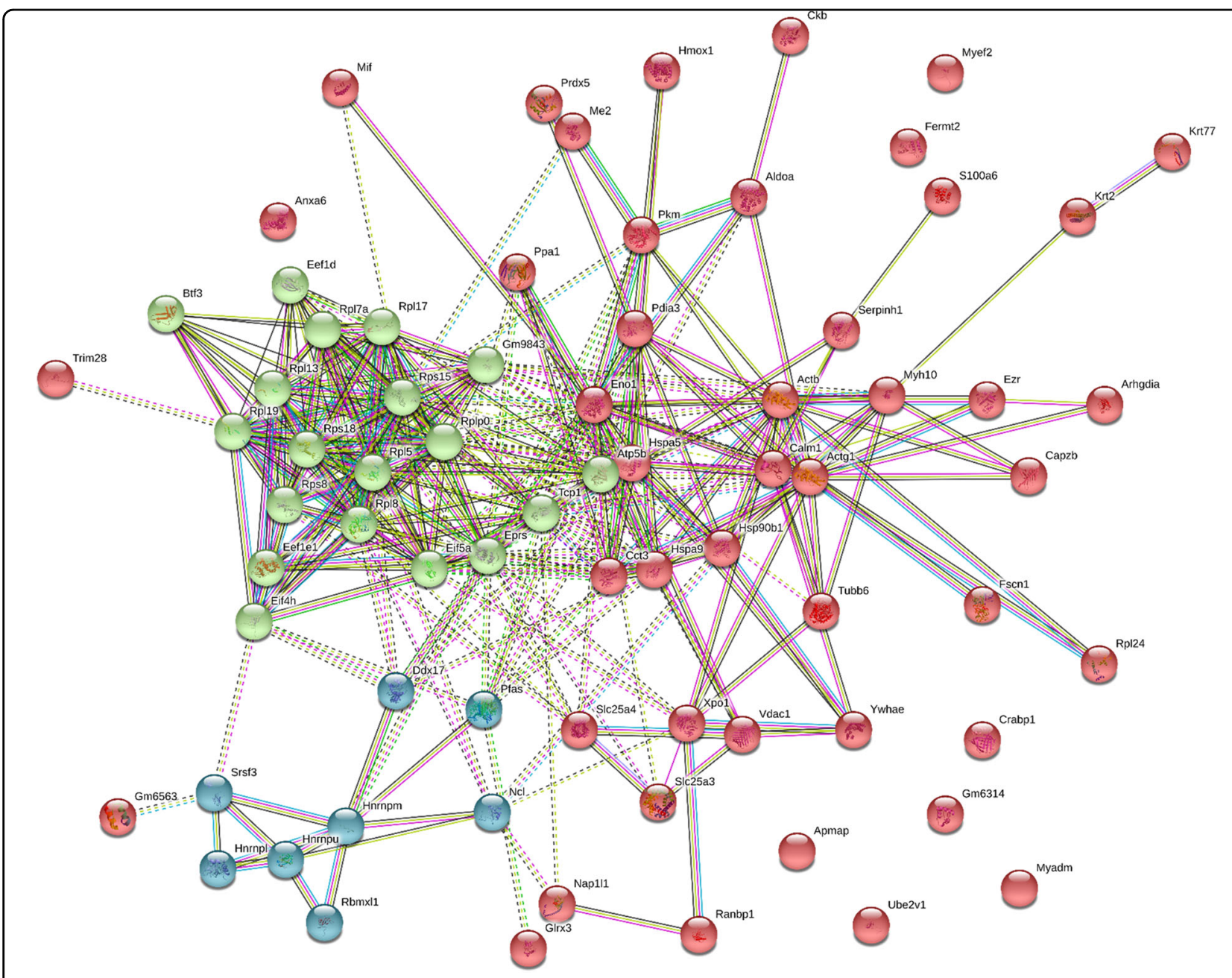

Fig. 8 Analysis of possible protein-protein interaction network using STRING tool. List of differentially expressed proteins was subjected to STRING analysis which found significantly more associations between proteins that would have occurred by chance $(p=1.0 \mathrm{e}-16)$. Three clusters detected by $k$-means algorithm are colored as follows: cluster 1 (46 proteins, red), proteins related to myelin sheet and extracellular exosomes; cluster 2 (19 proteins, green), proteins related to translation and ribosome; cluster 3 (8 proteins, blue), proteins related to nuclear ribonucleoprotein complex and splicing. For related lists of proteins see also Supplementary Table 5

primary $3 \mathrm{Tg}$ astrocytes ${ }^{40}$ and in 3Tg-iAstro lines, corroborating the $\mathrm{Ca}^{2+}$ imaging data presented in Fig. 4. Notably, none of iAstro lines responded to DHPG or glutamate (not shown), suggesting that function or/and expression of mGluR5 may be hampered by the immortalization process.

We used the iAstro model for proteomics to demonstrate its usefulness and to add information on the role of astrocytes in $\mathrm{AD}$. GO analysis of proteins down-regulated in 3Tg-iAstro as compared to WT-iAstro lines suggests that translation may be impaired in 3Tg-iAstro lines in two manners: (1) formation of nuclear ribonucleoprotein complex; and (2) formation of ribosomal multi-protein complex. Protein synthesis has already been suggested to be impaired in $\mathrm{AD}^{54-56}$ and recent findings suggest that it may occur early in $\mathrm{AD}$ pathogenesis ${ }^{57}$. In AD hippocampi, down-regulation of several proteins involved in chromatin compacting and regulation of rRNA transcription has been reported ${ }^{58}$ including nucleolin, which is also present in our list. Ribosomes are composed of the ribosomal RNAs and the ribosomal proteins that form the small subunit (40S) which binds to mRNA and the large subunit (60S) which binds to transfer RNAs and amino acids. Small subunit contains 33 proteins of which $3(9 \%)$ are present in our list of down-regulated proteins, while large subunit contains 46 proteins of which 8 (17.3\%) are present in our list, altogether suggesting that alterations in translation may represent an early astrocyte-specific event in $\mathrm{AD}$ pathogenesis ${ }^{57}$. Finally, we acknowledge that the straightforward translation of the results obtained on 
immortalized astrocytes in in vitro experiments to human $\mathrm{AD}$ pathogenesis is a rather speculative oversimplification. Further experiments, aiming at investigating the mechanistic aspects and confirmation of these results in vivo, are necessary to validate the presented data.

Transcriptome analysis of astrocytes freshly isolated from the brain of APPswe/PS1d9ex AD model mice at a symptomatic stage ${ }^{59}$, as well as of hippocampal cultured astrocytes from $3 \times \mathrm{Tg}$ - $\mathrm{AD}$ mice ${ }^{40}$, shows significant enrichment of genes in GO terms related to $\mathrm{Ca}^{2+}$. Proteomics data, however, do not reveal an overrepresentation of GO terms related to $\mathrm{Ca}^{2+}$. This may, at least in part, be explained by the fact that the shotgun mass spectrometry, used in this work, allows detection of only the most abundant proteins in iAstro lines. None of the proteins of the classical $\mathrm{Ca}^{2+}$ signaling toolkit, like $\mathrm{Ca}^{2+}$ transporters, channels or receptors, including P2Y2 purinergic receptor, has been detected. It is worth noting that the highest up-regulated protein in our analysis is calmodulin (CaM) (Table 1), whose role is to sense fluctuation of $\mathrm{Ca}^{2+}$ concentrations ${ }^{60,61}$. Downstream events include either transmission of the information to $\mathrm{Ca}^{2+}$-regulated signaling hubs, like $\mathrm{Ca}^{2}$ ${ }^{+} / \mathrm{CaM}$-activated kinases (CaMKs) of phosphatase calcineurin, or direct modulation of the activity of proteins, e.g., plasma membrane $\mathrm{Ca}^{2+}$ ATPase. Therefore, it is reasonable to suggest that 3.6-fold CaM overexpression in 3Tg-iAstro as compared to WT-iAstro (Table 1) may alter the entire $\mathrm{Ca}^{2+}$-dependent cellular homeostasis. While characterization of CaM-dependent processes in iAstro lines lies beyond the scope of present work, it is worth noting that in AD-related research, CaMactivated enzymes like CaMKII or calcineurin have been recurrently mentioned as central elements of disease pathogenesis and represent possible pharmacological targets ${ }^{41,62,63}$. Last, it has been recently suggested that $\mathrm{CaM}$ binds with high affinity to $A \beta$, thus representing a direct target for toxic $A \beta$ peptide ${ }^{64}$. In light of our observations, our data warrant further detailed examination of the role of $\mathrm{CaM}$ in $\mathrm{AD}$.

In conclusion, we have immortalized and characterized hippocampal astrocytes from WT and 3xTg-AD mouse pups. Using complementary methodologies we show that iAstro lines retain astroglial pattern of protein expression, retain fundamental astroglial housekeeping functions and show transcriptional and functional alterations found previously in primary astrocytes from $3 \times \mathrm{Tg}-\mathrm{AD}$ mice compared to WT mice. In addition, proteomic analysis suggests that protein synthesis, due to impaired nuclear RNA binding and alterations in ribosome composition, may be specifically impaired in AD astrocytes. Altogether, our results suggest that $3 \mathrm{Tg}$-iAstro may be a useful tool to study astrocyte-related alterations in AD.

\section{Materials and methods \\ Animals}

3xTg-AD mice used in this work were introduced by Frank LaFerla, Salvatore Oddo and colleagues in $2003^{45}$. These mice were developed on the mixed 129/C57BL6 background bearing knock-in mutation $\mathrm{PS}_{\mathrm{M} 146 \mathrm{~V}}{ }^{65}$ and in which $\mathrm{APP}_{\text {swe }}$ and $\mathrm{Tau}_{\mathrm{P} 301 \mathrm{~L}}$ transgenes were introduced. The 3xTg-AD animals show major histological hallmarks of $\mathrm{AD}$ represented by senile plaques and neurofibrillary tangles. These mice show progressive learning and memory deficit beginning from 4 months of age ${ }^{66}$. The $3 \times T g-A D$ mice and their respective non-transgenic controls (WT) ${ }^{45}$ were housed in the animal facility of the Università del Piemonte Orientale, were kept at three to four per cage and had unlimited access to water and food. Animals were managed in accordance with the European directive 2010/63/UE and with Italian law D.l. 26/2014. The procedures were approved by the local animal-health and ethical committee (Università del Piemonte Orientale) and were authorized by the national authority (Istituto Superiore di Sanità; authorization number N. 22/ 2013). All efforts were made to reduce the number of animals by following the $3 \mathrm{R}$ (replacement, reduction and refinement) rule.

\section{Primary astroglial cultures preparation and astrocyte purification}

For primary astroglial cultures, WT and 3xTg-AD P0P2 pups were killed by decapitation. Hippocampi were rapidly dissected and minced with a scalpel blade in cold calcium- and magnesium-free Hank's Balance Salt Solution (Sigma-Aldrich). The tissues were then digested with trypsin (Sigma-Aldrich; $0.25 \%, 37^{\circ} \mathrm{C}$ ) and triturated with 30 strokes of an automatic pipette. Non-dissociated tissue was allowed to sediment for $2 \mathrm{~min}$ and cell suspension was centrifuged for $5 \mathrm{~min}(200 \times g)$, resuspended in complete culture medium (Dulbecco's modified Eagle's medium (DMEM; Sigma-Aldrich, Cat. No. D5671) supplemented with $10 \%$ fetal bovine serum (Gibco, Cat. No. 10270), $2 \mathrm{mM}$ L-glutamine (Sigma-Aldrich), and $1 \%$ penicillin/streptomycin solution (Sigma-Aldrich) and plated in $60 \mathrm{~mm}$ Petri dishes (Falcon) (hippocampi from 3-6 pups per dish). Cells were maintained in a $5 \% \mathrm{CO}_{2} 37$ ${ }^{\circ} \mathrm{C}$ incubator. At $\sim 90 \%$ of confluence, cells were detached and microglial cells were removed by MACS using antiCD11b-conjugated beads (Miltenyi Biotech, Cat. No. 130093-634). Purified astrocytes were collected, resuspended in complete culture medium and plated in a $35 \mathrm{~mm}$ dish for immortalization.

\section{Production of SV40-containing replication-defective retroviral vectors}

Phoenix cells producing a replication-defective retrovirus $^{67}$, grown in $100 \mathrm{~mm}$ culture dishes (Falcon, $1.5 \times 10^{6}$ 
cells per dish), were transfected with pBABE-neo encoding neomycin phosphotransferase and SV40 large $\mathrm{T}$ antigen (Addgene, plasmid ID $1780^{68}$ ), using Lipofectamine 2000 reagent (Life Technologies, Segrate, Italy), according to the manufacturer's instructions. At $48 \mathrm{~h}$ after transfection, cell medium containing the retroviral vectors was collected and filtered through $0.4 \mu \mathrm{m}$ filters. The retroviral particles were precipitated by polyethylene glycol $\left(\mathrm{O} / \mathrm{N}, 4^{\circ} \mathrm{C}\right)$ prepared as described elsewhere ${ }^{69}$. The precipitate was concentrated by centrifugation $\left(3500 \times g, 30 \mathrm{~min}, 4^{\circ} \mathrm{C}\right)$, the supernatant was discarded and the pellet was resuspended in complete culture medium $(200 \mu \mathrm{l}$ per $8 \mathrm{ml}$ of initial medium), divided in $100 \mu \mathrm{l}$ aliquots and stored at $-80^{\circ} \mathrm{C}$.

\section{Astrocyte immortalization}

MACS-purified hippocampal astrocytes were plated in $35 \mathrm{~mm}$ culture dishes $\left(2.5 \times 10^{5}\right.$ cells per dish $)$ and $24 \mathrm{~h}$ later were transduced with retrovirus expressing SV40 large $\mathrm{T}$ antigen. To increase the infection efficiency, dishes were shaken $(80 \mathrm{rpm})$ overnight in a cell incubator. After $24 \mathrm{~h}$, fresh medium was added for $72 \mathrm{~h}$ and then replaced with medium containing $0.4 \mathrm{mg} / \mathrm{ml} \mathrm{G} 418$ disulfate salt solution (Sigma-Aldrich) ${ }^{70}$. At 3 to 4 weeks after cell selection, surviving cells were first sub-cultured in $100 \mathrm{~mm}$ dishes, expanded and maintained in complete culture medium supplemented with $0.4 \mathrm{mg} / \mathrm{ml} \mathrm{G} 418$ until passage 10. After thawing from cryopreservation, iAstro lines were maintained in complete culture medium without G418 and used for experiments between passages 12 and 20.

\section{Immunofluorescence}

WT- and 3Tg-iAstro cells, grown on $13 \mathrm{~mm}$ glass coverslips, were fixed in $4 \%$ formaldehyde, permeabilized (7 min in $0.1 \%$ Triton X-100 in phosphate-buffered saline (PBS)) and immunoprobed with an appropriate primary antibody (diluted in PBS supplemented with $1 \%$ gelatin) for $1 \mathrm{~h}$ at $37^{\circ} \mathrm{C}$. After 3 times washing in PBS, an Alexaconjugated secondary antibody (1:300 in PBS supplemented with $1 \%$ gelatin) was applied for $1 \mathrm{~h}$ at room temperature (RT). The following primary antibodies were used: AQP4 (Alomone Labs, Cat. No. 249-323), Aldh1l1 (Abcam, Cat. No. Ab190298), GS (Abcam, Cat. No. Ab73593), GFAP (Chemicon International, Cat. No. CBL411) and GLT-1 (Alomone labs, Cat. No. AGC-022). Secondary antibodies were as follows: Alexa Fluor 488 anti-mouse IgG, Alexa Fluor 555 anti-rabbit IgG (all secondary antibodies were from Molecular Probes, Life Technologies, Monza, Italy). Nuclei were counter-stained with 4',6-diamidino-2-phenylindole (DAPI). Images were acquired by Zeiss 710 confocal laser scanning microscope equipped with EC Plan-Neofluar 40×/1.30 Oil DIC M27 objective and Zen software.

\section{Total RNA extraction and real-time PCR}

Total RNA was extracted from $1 \times 10^{6}$ cells using Absolutely RNA miRNA kit (Agilent, Santa Clara, CA) according to the manufacturer's instructions. Total RNA $(0.5-1 \mu \mathrm{g})$ was retro-transcribed using random hexamers and ImProm-II RT system (Promega, Milan, Italy). Realtime PCR was performed using iTaq qPCR master mix according to the manufacturer's instructions (Bio-Rad, Segrate, Italy) on a SFX96 Real-time system (Bio-Rad). To normalize raw real-time PCR data, S18 ribosomal subunit was used. Sequences of oligonucleotide primers are provided in Supplementary Materials. The real-time PCR data are expressed as delta- $C(t)$ of gene of interest to $\mathrm{S} 18$ allowing appreciation of the relative expression level of a single gene.

\section{Electrophysiological recordings}

To perform patch-clamp experiments in whole-cell configuration, both WT-iAstro and 3Tg-iAstro were plated separately in $35 \mathrm{~mm}$ dishes $24 \mathrm{~h}$ prior experiments. Cells were plated at low density to allow recordings from isolated astrocytes. They were transferred from culture medium to an extracellular solution containing (in $\mathrm{mM}$ ): $138 \mathrm{NaCl}, 4 \mathrm{KCl}, 2 \mathrm{CaCl}_{2}, 1 \mathrm{MgCl}_{2}, 10$ glucose and 10 HEPES at pH 7.25 adjusted with $\mathrm{NaOH}$. Borosilicate patch pipettes were pulled with a P-1000 puller (Sutter Instruments, USA) and were filled with a solution containing (in mM): $140 \mathrm{KCl}, 2 \mathrm{NaCl}, 5 \mathrm{EGTA}, 0.5 \mathrm{CaCl}_{2}$ and 10 HEPES at pH 7.25 adjusted with $\mathrm{KOH}$. Pipette tip resistance containing this solution was between 3 and 5 $\mathrm{M} \Omega$. Experiments were performed using an EPC7 Plus amplifier (HEKA Elektronik, Germany) in voltage-clamp configuration (holding potential, $-80 \mathrm{mV}$ ). Access resistance (8-12 M $\Omega$ ) was compensated (80-90\%) and experiments were performed at $\mathrm{RT}$ and in a static bath. Data were acquired at $5 \mathrm{kHz}$ and filtered at $1 \mathrm{kHz}$ using a 7-pole Bessel filter and digitized with a low noise data acquisition system, Digidata 1440A (Molecular Devices, USA). Data were recorded and analyzed in pClamp 10 (Molecular Devices, Crisel Instruments, Italy). Data were initially processed with Microsoft Excel. Plots, bar diagrams and figure preparations were finalized with GraphPad Prism (GraphPad Software, La Jolla, CA).

\section{Cell lysates}

WT- and 3Tg-iAstro cells were plated at a density of $2 \times$ $10^{5}$ cells $/ 100 \mathrm{~mm}$ dish and cultured until $80-90 \%$ of confluence. Then, cells were washed twice with cold PBS and lysed in $800 \mu \mathrm{l}$ lysis buffer $(50 \mathrm{mM}$ Tris- $\mathrm{HCl} \mathrm{pH}$ 7.4, 150 $\mathrm{mM} \mathrm{NaCl}, 0,5 \mathrm{mM}$ EDTA, 0,1\% NP40, 0,1\% SDS) supplemented with protease and phosphatase inhibitors cocktail (Thermo Scientific Halt Protease and Phosphatase Inhibitor Cocktail, Cat. No. 78444). For each sample, protein concentration was measured by BCA assay (Quanti Pro BCA 
Asssay Kit, SIGMA, Cat. No. QPBCA-1Kt) and read by BioRad SMART ${ }^{\mathrm{TM}}$ Plus Spectrophotometer.

\section{Western blotting}

Specific astroglial proteins were detected in WT- and 3Tg-iAstro lysates by western blotting (WB). Each sample, containing $100 \mu \mathrm{g}$ of protein, was diluted $1: 1$ in $2 \times$ Laemmli sample buffer, heated at $95^{\circ} \mathrm{C}$ for $5 \mathrm{~min}$, then resolved by sodium dodecyl sulfate-polyacrylamide gel electrophoresis (SDS-PAGE). Proteins were electrophoretically transferred to nitrocellulose membranes and the membranes were blocked for $1 \mathrm{~h}$ in $5 \%(\mathrm{w} / \mathrm{v})$ non-fat dry milk in Tris-buffered saline containing Tween-20. After incubation with appropriate primary and secondary antibodies, signals were revealed using enhanced chemiluminescence (Super Signal West Femto Maximum Sensitivity Substrate, Thermo Scientific, Cat. No. 34095), and were visualized by a Bio-Rad ChemiDoc touch Imaging system. Anti-AQP4 (1:1000, Alomone Labs, Cat. 249323), anti-GS (1:2000, Abcam, Cat. No. Ab73593), antiAldh111 (1:2000, Abcam, Cat. No. Ab190298) and anti- $\beta$ actin (1:10,000, Sigma, Cat. No. A1978) antibodies were used to develop western blots, as indicated in the figure legends. Densitometric analysis was performed using Quantity One software and is expressed as mean \pm SEM from at least 3 independent runs. Analysis of variance (ANOVA) test was used for statistical analysis. Note relatively big variability between technical replicates in WB analysis, which may be explained by low level of specific astroglial proteins expressed in iAstro lines.

\section{Fura-2 calcium imaging}

For $\mathrm{Ca}^{2+}$ imaging, iAstro lines grown onto $24 \mathrm{~mm}$ round coverslips were loaded with Fura-2/AM (Life Technologies, Milan, Italy, Cat. No. F1201) in the presence of $0.005 \%$ Pluronic F-127 (Life Technologies, Cat. No. P6867) and $10 \mu \mathrm{M}$ sulfinpyrazone (Sigma, Cat. No. S9509) in KRB solution ( $125 \mathrm{mM} \mathrm{NaCl}, 5 \mathrm{mM} \mathrm{KCl}, 1 \mathrm{mM}$ $\mathrm{Na}_{3} \mathrm{PO}_{4}, 1 \mathrm{mM} \mathrm{MgSO}$, $5.5 \mathrm{mM}$ glucose, $20 \mathrm{mM}$ HEPES, $\mathrm{pH}$ 7.4) supplemented with $2 \mathrm{mM} \mathrm{CaCl}$. After loading and $30 \mathrm{~min}$ of de-esterification, the coverslips were mounted in an acquisition chamber on the stage of a Leica epifluorescence microscope equipped with a S Fluor $40 \times$ / 1.3 objective. Cells were alternatively excited at 340/380 $\mathrm{nm}$ by the monochromator Polichrome V (Till Photonics, Munich, Germany) and the fluorescent signal was collected by a CCD camera (Hamamatsu, Japan) through bandpass $510 \mathrm{~nm}$ filter; the experiments were controlled and images analyzed with MetaFluor (Molecular Devices, Sunnyvale, CA, USA) software. The cells were stimulated by $20 \mu \mathrm{M}$ ATP. To quantify the difference in the amplitude of $\mathrm{Ca}^{2+}$ transients, the ratio values were normalized according to the formula $(\Delta \mathrm{F}) / \mathrm{F}_{0}$ (referred to as norm. Fura ratio).

\section{Proteomic analysis \\ In solution digestion}

Cell lysates were digested using the following protocol: samples were prepared to have $100 \mu \mathrm{g}$ of protein in a final volume of $25 \mu \mathrm{l}$ of $100 \mathrm{mM} \mathrm{NH} \mathrm{NCO}_{3}$. Proteins were reduced using $2.5 \mu \mathrm{l}$ of dithiothreitol (200 mM DTT stock solution) (Sigma) at $90^{\circ} \mathrm{C}$ for $20 \mathrm{~min}$, and alkylated with $10 \mu \mathrm{l}$ of Cysteine Blocking Reagent (iodoacetamide (IAM), $200 \mathrm{mM}$ Sigma) for $1 \mathrm{~h}$ at room temperature in the dark. DTT stock solution was then added to destroy the excess of IAM. After dilution with $300 \mu \mathrm{l}$ of water and $100 \mu \mathrm{l}$ of $\mathrm{NH}_{4} \mathrm{HCO}_{3}$ to raise $\mathrm{pH}$ 7.5-8.0, $5 \mu \mathrm{g}$ of trypsin (Promega, Sequence Grade) was added and digestion was performed overnight at $37^{\circ} \mathrm{C}$. Trypsin activity was stopped by adding $2 \mu \mathrm{l}$ of neat formic acid and samples were dried by Speed Vacuum $^{71}$.

The peptide digests were desalted on the Discovery ${ }^{\circledR}$ DSC-18 solid-phase extraction (SPE) 96-well Plate (25 $\mathrm{mg} /$ well) (Sigma-Aldrich Inc., St. Louis, MO, USA). The SPE plate was preconditioned with $1 \mathrm{ml}$ of acetonitrile and $2 \mathrm{ml}$ of water. After the sample loading, the SPE was washed with $1 \mathrm{ml}$ of water. The adsorbed proteins were eluted with $800 \mu \mathrm{l}$ of acetonitrile/water (80:20). After desalting, samples were vacuum evaporated and reconstituted with $20 \mu \mathrm{l}$ of $0.05 \%$ formic acid in water. Then, 2 $\mu \mathrm{l}$ of stable-isotope-labeled peptide standard (DPEVRPTSAVAA, Val- $13 \mathrm{C} 515 \mathrm{~N} 1$ at V10, Cellmano Biotech Limited, Anhui, China) was spiked into the samples before the liquid chromatography-tandem mass spectrometry (LC-MS/MS) analysis and used for instrument quality control.

\section{Label-free proteomic analysis}

LC-MS/MS analyses were performed using a micro-LC Eksigent Technologies (Dublin, USA) system with a stationary phase of a Halo Fused C18 column $(0.5 \times 100 \mathrm{~mm}$, $2.7 \mu \mathrm{m}$; Eksigent Technologies, Dublin, USA). The injection volume was $4.0 \mu \mathrm{l}$ and the oven temperature was set at $40{ }^{\circ} \mathrm{C}$. The mobile phase was a mixture of $0.1 \%(\mathrm{v} / \mathrm{v})$ formic acid in water (A) and $0.1 \%(\mathrm{v} / \mathrm{v})$ formic acid in acetonitrile (B), eluting at a flow rate of $15.0 \mu \mathrm{l} / \mathrm{min}$ at an increasing concentration of solvent B from 2 to $40 \%$ in 30 min. The LC system was interfaced with a $5600+$ TripleTOF system (AB Sciex, Concord, Canada) equipped with a DuoSpray Ion Source and CDS (Calibrant Delivery System). Samples used to generate the SWATH-MS (sequential window acquisition of all theoretical mass spectra) spectral library were subjected to the traditional data-dependent acquisition (DDA): the mass spectrometer analysis was performed using a mass range of 100-1500 Da (TOF scan with an accumulation time of $0.25 \mathrm{~s}$ ), followed by a MS/MS product ion scan from 200 to $1250 \mathrm{Da}$ (accumulation time of $5.0 \mathrm{~ms}$ ) with the abundance threshold set at $30 \mathrm{cps}$ ( 35 candidate ions can 
be monitored during every cycle). Samples were then subjected to cyclic data-independent analysis (DIA) of the mass spectra, using a $25 \mathrm{Da}$ window. A $50 \mathrm{~ms}$ survey scan (TOF-MS) was performed, followed by MS/MS experiments on all precursors. These MS/MS experiments were performed in a cyclic manner using an accumulation time of $40 \mathrm{~ms}$ per $25 \mathrm{Da}$ swath (36 swaths in total) for a total cycle time of $1.5408 \mathrm{~s}$. The ions were fragmented for each MS/MS experiment in the collision cell using the rolling collision energy. The MS data were acquired with Analyst TF 1.7 (SCIEX, Concord, Canada). Three instrumental replicates for each sample were subjected to the DIA analysis $^{72,73}$.

\section{Protein database search}

The mass spectrometry files were searched using Protein Pilot (AB SCIEX, Concord, Canada) and Mascot (Matrix Science Inc., Boston, USA). Samples were input in the Protein Pilot software v. 4.2 (AB SCIEX, Concord, Canada), which employs the Paragon algorithm, with the following parameters: cysteine alkylation, digestion by trypsin, no special factors and false discovery rate (FDR) at $1 \%$. The UniProt Swiss-Prot reviewed database containing mouse proteins (version 20july15, containing 23,304 sequence entries). The Mascot search was performed on Mascot v. 2.4, the digestion enzyme selected was trypsin, with 2 missed cleavages and a search tolerance of $50 \mathrm{ppm}$ was specified for the peptide mass tolerance, and $0.1 \mathrm{Da}$ for the MS/MS tolerance. The charges of the peptides to search for were set to $2+, 3+$ and $4+$, and the search was set on monoisotopic mass. The instrument was set to ESI-QUAD-TOF (electrospray ionization quadrupole time-of-flight) and the following modifications were specified for the search: carbamidomethyl cysteines as fixed modification and oxidized methionine as variable modification.

\section{Protein quantification}

The quantification was performed by integrating the extracted ion chromatogram of all the unique ions for a given peptide. The quantification was carried out with PeakView 2.0 and MarkerView 1.2. (Sciex, Concord, ON, Canada). Six peptides per protein and six transitions per peptide were extracted from the SWATH files. Shared peptides were excluded as well as peptides with modifications. Peptides with FDR lower than $1.0 \%$ were exported in MarkerView for the $t$-test.

\section{Analysis of identified proteins}

For identification of the number of detected proteins for each genotype, the intersection of four lists, corresponding to four analyzed lines per genotype, was performed, yielding lists of common proteins detected in four WTand four 3Tg-iAstro lines (1119 and 1045, respectively).
The intersection of the latter two lists gave proteins detected in both WT- and 3Tg-iAstro cells (856 proteins).

\section{Gene ontology analysis}

GO analysis was performed using DAVID v.6.8 tool (https://david.ncifcrf.gov/) $)^{74}$. For the analysis of the overrepresented GO terms, for the background, a list containing all proteins detected in WT-iAstro and 3TgiAstro lines (1308 proteins) was used. Over-represented GO terms which passed Benjamini correction $(p<0.05)$ were considered significant. For prediction of protein-protein interactions and clustering using $k$ means algorithm, STRING v.10.5 online software was used (https://string-db.org//)

\section{Glutamate uptake}

Primary astrocytes, WT and 3Tg-iAstro lines were cultured in 24-well plates at $5 \times 10^{4}$ cells /well. Cells were treated in DMEM/F12 medium with $5 \mathrm{mM}$ glutamate (Lglutammic acid momosodium salt, Sigma, Cat. No. G1626) with or without TBOA ((3S)-3-[[3-[[4-(trifluoromethyl)bemzoyl]amino]phenyl]methoxy]-L-aspartic acid, Tocris, Cat. No. 2532). After $2 \mathrm{~h}$, supernatants were collected, centrifuged $\left(16,000 \times g\right.$ for $10 \mathrm{~min}$ at $\left.4{ }^{\circ} \mathrm{C}\right)$ and analyzed for residual glutamate using the protocol described by the manufacturers (Amplex ${ }^{\circledR}$ Red Glutamic Acid/Glutamate Oxidase Kit, Invitrogen, Cat. No. A12221). Glutamate uptake was calculated as difference of fluorescence at $590 \mathrm{~nm}$ between glutamate plus TBOA and glutamate alone and normalized to protein concentrations of corresponding cell lysate.

\section{LPS and TNFa treatment}

For treatment with bacterial LPS (lipopolysaccharides from Escherichia coli O111:B4, Sigma, Cat. No. L2630) and TNF $\alpha$ (Peprotech, London, UK, Cat. No. 300-01A), cells were plated in 12 -well plates $\left(1 \times 10^{5}\right.$ cells per well) and, upon confluence, were treated with either LPS (for 3 h) or TNF $($ for $6 \mathrm{~h}$ ). The cells were then lysed in $500 \mu \mathrm{l}$ Trizol Reagent (Life Technologies) and total RNA was extracted according to the manufacturer's instructions. First-strand complementary DNA and real-time PCR were performed as described above. Oligonucleotide primers for iNOS are listed in Supplementary Materials.

\section{Experimental groups selection and statistical analysis}

For the selection of experimental groups, the following criteria were adopted: in experiments in which entire populations of cells were analyzed (western blotting, realtime PCR and proteomic analysis), experimental group was composed of 4 independently generated iAstro lines for each genotype (WT-iAstro\#2, \#3, \#5 and \#6, and 3TgiAstro\#2, \#3, \#4 and \#6) representing biological replicates. Technical replicates consisted of at least three 
independent experiments of each iAstro line. In experiments, in which single-cell analysis was performed (immunocytochemistry, electrophysiology and Fura-2 $\mathrm{Ca}^{2}$ + imaging), experimental groups were composed of independent experiments (at least three coverslip (technical replicates) from at least three different experiments) of one line per genotype were used (WT-iAstro\#2 and 3Tg-iAstro\#2).

Statistical analysis was performed using GraphPad Prism software v.7. For analysis of real-time PCR and $\mathrm{Ca}^{2+}$ imaging data, a two-tailed unpaired Students's $t$-test was used. For western blot ANOVA on raw followed by Tukey's post-hoc test was used. Differences were considered significant at $p<0.05$.

\section{Acknowledgements}

This work had the following financial supports: grants 2013-0795 to P.L.C. and 2014-1094 to D.L. from the Fondazione Cariplo; grant 2016 to D.L. from The Università del Piemonte Orientale; and grant PRIN-2015N4FKJ4 to P.L.C. from the Italian Ministry of Education. L.T. was supported by fellowship from the CRT Foundation (1393-2017).

\section{Author details \\ 'Department of Pharmaceutical Sciences, Università degli Studi del Piemonte Orientale, Novara, Italy. ${ }^{2}$ Department of Sciences and Technological Innovation, Università degli Studi del Piemonte Orientale, Alessandria, Italy. ${ }^{3}$ ISALIT S.r.I., Spin-off of Università degli Studi del Piemonte Orientale, Novara, Italy. ${ }^{4}$ Department of Biomedical and Biotechnological Sciences, Section of Pharmacology, University of Catania, Via Santa Sofia, 97, 95123 Catania, Italy. ${ }^{5}$ Present address: International Center for T1D, Pediatric Clinic Research Center Fondazione Romeo ed Enrica Invernizzi, Department of Biomedical and Clinical Science L. Sacco, University of Milan, Milan, Italy}

\section{Conflict of interest}

The authors declare that they have no conflict of interest.

\section{Publisher's note}

Springer Nature remains neutral with regard to jurisdictional claims in published maps and institutional affiliations.

Supplementary Information accompanies this paper at (https://doi.org/ 10.1038/s41419-018-1264-8).

Received: 5 July 2018 Revised: 3 December 2018 Accepted: 5 December 2018

Published online: 10 January 2019

\section{References}

1. Rodríguez, J. J. et al. Complex and region-specific changes in astroglial markers in the aging brain. Neurobiol. Aging 35, 15-23 (2014).

2. Verkhratsky, A., Zorec, R., Rodríguez, J. J. \& Parpura, V. Astroglia dynamics in ageing and Alzheimer's disease. Curr. Opin. Pharmacol. 26, 74-79 (2016).

3. Fages, C., Khelil, M., Rolland, B., Bridoux, A. M. \& Tardy, M. Glutamine synthetase: a marker of an astroglial subpopulation in primary cultures of defined brain areas. Dev. Neurosci. 10, 47-56 (1988).

4. Hansson, E. Accumulation of putative amino acid neurotransmitters, monoamines and D-Ala2-Met-enkephalinamide in primary astroglial cultures from various brain areas, visualized by autoradiography. Brain Res. 289, 189-196 (1983).

5. Bernascone, S., Erriquez, J., Ferraro, M., Genazzani, A. A. \& Distasi, C. Novel adenosine and CAMP signalling pathways in migrating glial cells. Cell Calcium 48, 83-90 (2010).
6. Eichberg, J., Shein, H. M. \& Hauser, G. Lipid composition and metabolism of cultured hamster brain astrocytes. J. Neurochem. 27, 679-685 (1976).

7. Fresu, L., Dehpour, A., Genazzani, A. A., Carafoli, E. \& Guerini, D. Plasma membrane calcium ATPase isoforms in astrocytes. Glia 28, 150-155 (1999).

8. Nowak, L., Ascher, P. \& Berwald-Netter, Y. lonic channels in mouse astrocytes in culture. J. Neurosci. 7, 101-109 (1987).

9. Alliot, F. \& Pessac, B. Astrocytic cell clones derived from established cultures of 8-day postnatal mouse cerebella. Brain Res. 306, 283-291 (1984).

10. An, K. et al. Subarachnoid transplantation of immortalized galaninoverexpressing astrocytes attenuates chronic neuropathic pain. Eur. J. Pain 14, 595-601 (2010).

11. Behrstock, S. P., Anantharam, V., Thompson, K. W., Schweitzer, E. S. \& Tobin, A. J. Conditionally-immortalized astrocytic cell line expresses GAD and secretes GABA under tetracycline regulation. J. Neurosci. Res. 60, 302-310 (2000).

12. Claassen, D. A. \& Lahue, R. S. Expansions of CAG.CTG repeats in immortalized human astrocytes. Hum. Mol. Genet. 16, 3088-3096 (2007).

13. Dai, B. et al. FoxM1B regulates NEDD4-1 expression, leading to cellular transformation and full malignant phenotype in immortalized human astrocytes. Cancer Res. 70, 2951-2961 (2010).

14. Frisa, P. S. \& Jacobberger, J. W. Cell density related gene expression: SV40 large T antigen levels in immortalized astrocyte lines. BMC Cell Biol. 3, 10 (2002).

15. Furihata, T., Ito, R., Kamiichi, A., Saito, K. \& Chiba, K. Establishment and characterization of a new conditionally immortalized human astrocyte cell line. J. Neurochem. 136, 92-105 (2016).

16. Major, E. O. et al. Establishment of a line of human fetal glial cells that supports JC virus multiplication. Proc. Natl Acad. Sci. USA 82, 1257-1261 (1985).

17. Morikawa, M. et al. Isolation and characterization of a new immortal rat astrocyte with a high expression of NGF mRNA. Neurosci. Res. 39, 205-212 (2001).

18. Morikawa, M. et al. Production and characterization of astrocyte-derived human apolipoprotein $\mathrm{E}$ isoforms from immortalized astrocytes and their interactions with amyloid-beta. Neurobiol. Dis. 19, 66-76 (2005).

19. Radany, E. H. et al. Directed establishment of rat brain cell lines with the phenotypic characteristics of type 1 astrocytes. Proc. Natl Acad. Sci. USA 89, 6467-6471 (1992)

20. Raofi, S., Wong, P. K. \& Wilcox, R. E. Modulation of G-protein linked CAMP accumulation in immortalized murine cortical astrocytes by retroviral infection. Brain Res. 862, 230-233 (2000).

21. Roy, N. S. et al. Functional engraftment of human ES cell-derived dopaminergic neurons enriched by coculture with telomerase-immortalized midbrain astrocytes. Nat. Med. 12, 1259-1268 (2006).

22. Sacchettoni, S. A., Benchaibi, M., Sindou, M., Belin, M. F. \& Jacquemont, B. Glutamate-modulated production of GABA in immortalized astrocytes transduced by a glutamic acid decarboxylase-expressing retrovirus. Glia 22, 86-93 (1998).

23. Xu, Y., Tian, X., An, K., Yang, H. \& Tian, Y. Lumbar transplantation of immortalized enkephalin-expressing astrocytes attenuates chronic neuropathic pain. Eur. J. Pain. 12, 525-533 (2008)

24. Bellot-Saez, A., Kékesi, O., Morley, J. W. \& Buskila, Y. Astrocytic modulation of neuronal excitability through $\mathrm{K}+$ spatial buffering. Neurosci. Biobehav. Rev. 77, 87-97 (2017)

25. Chisari, M. et al. Purinergic P2Y1 receptors control rapid expression of plasma membrane processes in hippocampal astrocytes. Mol. Neurobiol. 54 4081-4093 (2017)

26. Milner, R. Astrocytes: Methods and Protocols (Humana Press, New York, 2012).

27. Robinson, M. B. \& Jackson, J. G. Astroglial glutamate transporters coordinate excitatory signaling and brain energetics. Neurochem. Int. 98, 56-71 (2016).

28. Rose, C. R. et al. Astroglial glutamate signaling and uptake in the hippocampus. Front. Mol. Neurosci. 10, 451 (2017).

29. Khakh, B. S. \& McCarthy, K. D. Astrocyte calcium signaling: from observations to functions and the challenges therein. Cold Spring Harb. Perspect. Biol. 7, a020404 (2015)

30. Verkhratsky, A. Glial calcium signaling in physiology and pathophysiology. Acta Pharmacol. Sin. 27, 773-780 (2006).

31. Lim, D., Rocchio, F., Lisa, M. \& Fcancesco, M. From pathology to physiology of calcineurin signalling in astrocytes. Opera Med. Physiol. 2, 122-140 (2016).

32. Lim, D. et al. Amyloid beta deregulates astroglial mGluR5-mediated calcium signaling via calcineurin and Nf-kB. Glia 61, 1134-1145 (2013).

33. Grolla, A. A. et al. A $\beta$ leads to $\mathrm{Ca}^{2+}$ signaling alterations and transcriptional changes in glial cells. Neurobiol. Aging 34, 511-522 (2013). 
34. Grolla, A. A. et al. Amyloid- $\beta$ and Alzheimer's disease type pathology differentially affects the calcium signalling toolkit in astrocytes from different brain regions. Cell Death Dis. 4, e623 (2013).

35. Alberdi, E. et al. $\mathrm{Ca}(2+)$-dependent endoplasmic reticulum stress correlates with astrogliosis in oligomeric amyloid $\beta$-treated astrocytes and in a model of Alzheimer's disease. Aging Cell. 12, 292-302 (2013).

36. Casley, C. S. et al. Up-regulation of astrocyte metabotropic glutamate receptor 5 by amyloid- $\beta$ peptide. Brain Res. 1260, 65-75 (2009).

37. Delekate, A. et al. Metabotropic P2Y1 receptor signalling mediates astrocytic hyperactivity in vivo in an Alzheimer's disease mouse model. Nat. Commun. 5 $5422(2014)$

38. Kuchibhotla, K. V., Lattarulo, C. R., Hyman, B. T. \& Bacskai, B. J. Synchronous hyperactivity and intercellular calcium waves in astrocytes in Alzheimer mice. Science 323, 1211-1215 (2009).

39. Ronco, $\mathrm{V}$. et al. Differential deregulation of astrocytic calcium signalling by amyloid- $\beta$, TNFa, IL-1 $\beta$ and LPS. Cell Calcium 55, 219-229 (2014).

40. Ruffinatti, F. et al. Transcriptional remodeling in primary hippocampal astrocytes from an Alzheimer's disease mouse model. Curr. Alzheimer Res. 15 986-1004(2018)

41. Furman, J. L. \& Norris, C. M. Calcineurin and glial signaling: neuroinflammation and beyond. J. Neuroinflamm. 11, 158 (2014).

42. Tapella, L. et al. TGF- $\beta 2$ and TGF- $\beta 3$ from cultured $\beta$-amyloid-treated or 3xTgAD-derived astrocytes may mediate astrocyte-neuron communication. Eur. J. Neurosci. 47, 211-221 (2018).

43. Hanrieder, J., Wicher, G., Bergquist, J., Andersson, M. \& Fex-Svenningsen, A. MALDI mass spectrometry based molecular phenotyping of CNS glial cells for prediction in mammalian brain tissue. Anal. Bioanal. Chem. 401, 135-147 (2011).

44. Yang, J.-W., Suder, P., Silberring, J. \& Lubec, G. Proteome analysis of mouse primary astrocytes. Neurochem. Int. 47, 159-172 (2005).

45. Oddo, S. et al. Triple-transgenic model of Alzheimer's disease with plaques and tangles: intracellular Abeta and synaptic dysfunction. Neuron 39, 409-421 (2003).

46. Hubbard, K. \& Ozer, H. L. Mechanism of immortalization. Age (Omaha) 22 65-69 (1999).

47. Schildknecht, S. et al. Characterization of mouse cell line IMA 2.1 as a potential model system to study astrocyte functions. ALTEX 29, 261-274 (2012).

48. Bradford, R., Koppel, H., Pilkington, G. J., Thomas, D. G. \& Darling, J. L. Heterogeneity of chemosensitivity in six clonal cell lines derived from a spontaneous murine astrocytoma and its relationship to genotypic and phenotypic characteristics. J. Neurooncol. 34, 247-261 (1997).

49. Hastings, R. J. \& Franks, L. M. Cellular heterogeneity in a tissue culture cell line derived from a human bladder carcinoma. Br. J. Cancer 47, 233-244 (1983).

50. Stockholm, D. et al. The origin of phenotypic heterogeneity in a clonal cell population in vitro. PLOS ONE 2, e394 (2007).

51. Lim, D. et al. Calcium signalling toolkits in astrocytes and spatiotemporal progression of Alzheimer's disease. Curr. Alzheimer Res. 13, 359-369 (2016)

52. Lim, D., Ronco, V., Grolla, A. A., Verkhratsky, A. \& Genazzani, A. A. Glial calcium signalling in Alzheimer's disease. Rev. Physiol. Biochem. Pharmacol. 167, 45-65 (2014).

53. Vincent, A. J., Gasperini, R., Foa, L. \& Small, D. H. Astrocytes in Alzheimer's disease: emerging roles in calcium dysregulation and synaptic plasticity. J. Alzheimers Dis. 22, 699-714 (2010).

54. Dönmez-Altuntaş, $H$. et al. Evaluation of the nucleolar organizer regions in Alzheimer's disease. Gerontology 51, 297-301 (2005).

55. Langstrom, N. S., Anderson, J. P., Lindroos, H. G., Winblad, B. \& Wallace, W. C. Alzheimer's disease-associated reduction of polysomal mRNA translation. Brain Res. Mol. Brain. Res. 5, 259-269 (1989).
56. Sajdel-Sulkowska, E. M. \& Marotta, C. A. Alzheimer's disease brain: alterations in RNA levels and in a ribonuclease-inhibitor complex. Science 225, 947-949 (1984).

57. Ding, Q., Markesbery, W. R., Chen, Q., Li, F. \& Keller, J. N. Ribosome dysfunction is an early event in Alzheimer's disease. J. Neurosci. 25, 9171-9175 (2005).

58. Hernández-Ortega, K., Garcia-Esparcia, P., Gil, L., Lucas, J. J. \& Ferrer, I. Altered machinery of protein synthesis in Alzheimer's: from the nucleolus to the ribosome. Brain Pathol. 26, 593-605 (2016).

59. Orre, M. et al. Acute isolation and transcriptome characterization of cortical astrocytes and microglia from young and aged mice. Neurobiol. Aging 35, 1-14 (2014)

60. Krebs, J. A survey of structural studies on calmodulin. Cell Calcium 2, 295-311 (1981).

61. Means, A. R. \& Dedman, J. R. Calmodulin--an intracellular calcium receptor. Nature 285, 73-77 (1980).

62. Popugaeva, E., Pchitskaya, E. \& Bezprozvanny, I. Dysregulation of neuronal calcium homeostasis in Alzheimer's disease - a therapeutic opportunity? Biochem. Biophys. Res. Commun. 483, 998-1004 (2017).

63. Reese, L. C., Laezza, F., Woltjer, R. \& Taglialatela, G. Dysregulated phosphorylation of $\mathrm{Ca}(2+)$ /calmodulin-dependent protein kinase II- $a$ in the hippocampus of subjects with mild cognitive impairment and Alzheimer's disease. J. Neurochem. 119, 791-804 (2011)

64. Corbacho, I., Berrocal, M., Török, K., Mata, A. M. \& Gutierrez-Merino, C High affinity binding of amyloid $\beta$-peptide to calmodulin: Structural and functional implications. Biochem. Biophys. Res. Commun. 486, 992-997 (2017).

65. Guo, Q. et al. Increased vulnerability of hippocampal neurons to excitotoxic necrosis in presenilin-1 mutant knock-in mice. Nat. Med. $\mathbf{5}$ 101-106 (1999).

66. Billings, L. M., Oddo, S., Green, K. N., McGaugh, J. L. \& LaFerla, F. M. Intraneuronal Abeta causes the onset of early Alzheimer's disease-related cognitive deficits in transgenic mice. Neuron 45, 675-688 (2005).

67. Mann, R., Mulligan, R. C. \& Baltimore, D. Construction of a retrovirus packaging mutant and its use to produce helper-free defective retrovirus. Cell 33, 153-159 (1983).

68. Hahn, W. C. et al. Enumeration of the simian virus 40 early region elements necessary for human cell transformation. Mol. Cell. Biol. 22, 2111-2123 (2002)

69. Lim, D., Bertoli, A., Sorgato, M. C. \& Moccia, F. Generation and usage of aequorin lentiviral vectors for $\mathrm{Ca}(2+)$ measurement in sub-cellular compartments of hard-to-transfect cells. Cell Calcium 59, 228-239 (2016).

70. Frisa, P. S., Goodman, M. N., Smith, G. M., Silver, J. \& Jacobberger, J. W. Immortalization of immature and mature mouse astrocytes with SV40 T antigen. J. Neurosci. Res. 39, 47-56 (1994).

71. Martinotti, S. et al. HMGB1 osteo-modulatory action on osteosarcoma SaOS-2 cell line: an integrated study from biochemical and -omics approaches. J. Cell. Biochem. 117, 2559-2569 (2016).

72. Cvijetic, S. et al. Cell autonomous and noncell-autonomous role of NF-kB p50 in astrocyte-mediated fate specification of adult neural progenitor cells. Glia 65, 169-181 (2017).

73. Albanese, P. et al. Isolation of novel PSII-LHCII megacomplexes from pea plants characterized by a combination of proteomics and electron microscopy. Photo. Res. 130, 19-31 (2016).

74. Huang, D. W., Sherman, B. T. \& Lempicki, R. A. Systematic and integrative analysis of large gene lists using DAVID bioinformatics resources. Nat. Protoc. $\mathbf{4}$ 44-57 (2009).

75. Szklarczyk, D. et al. The STRING database in 2017: quality-controlled proteinprotein association networks, made broadly accessible. Nucleic Acids Res. $\mathbf{4 5}$ D362-D368 (2017). 